\title{
Dynamics of the quark-antiquark interaction and the universality of Regge trajectories
}

\author{
A. M. Badalian ${ }^{\dagger}$ \\ Institute of Theoretical and Experimental Physics, 117218 Moscow, Russia \\ B. L. G. Bakker* \\ Faculty of Science, Vrije Universiteit, De Boelelaan 1081, 1081HV Amsterdam, Netherlands
}

(Received 22 February 2019; published 9 August 2019)

\begin{abstract}
The dynamical picture of a quark-antiquark interaction in light mesons, which provides linearity of radial and orbital Regge trajectories (RT), is studied with the use of the relativistic string Hamiltonian with flattened confining potential (CP) and taking into account three negative corrections: the gluon-exchange, the selfenergy, and the string corrections. Due to the flattening effect the radial slope $\beta_{n}$ and the orbital slope $\beta_{l}$ of the Regge trajectories decrease by $\sim 30 \%$ as compared to those in linear $\mathrm{CP}$, while the string correction decreases only the orbital slope by the value $\sim 10 \%$. The self-energy correction is very important and has large magnitude, $\sim-300 \mathrm{MeV}$ for high excitations. It also provides the linearity of the RT, built for the centroid squared masses, and gives a small value of the intercept, $\beta_{0}=0.50(1) \mathrm{GeV}^{2}$, equal to the squared centroid mass of $\rho(1 S)$. If the universal gluon-exchange potential without fitting parameters and screening function, as in heavy quarkonia, is taken, then the radial slope, $\beta_{n}=1.15(9) \mathrm{GeV}^{2}(l \neq 0)$, and the orbital slope, $\beta_{l}=1.03(9) \mathrm{GeV}^{2}$, have close values and the RT can be considered as approximately universal.
\end{abstract}

DOI: 10.1103/PhysRevD.100.034010

\section{INTRODUCTION}

The spectroscopy of light mesons refers to the field where nonperturbative QCD dominates and the Regge trajectories (RT), both orbital and radial, appear to be the most explicit manifestation of nonperturbative effects. It is known that the leading RT in the $\left(M^{2}, J\right)$-plane has a linear behavior with the slope $\beta_{J}(\exp )=2 \pi \sigma=1.13(1) \mathrm{GeV}^{2}$, which corresponds to the value of the string tension $\sigma=0.180(2) \mathrm{GeV}^{2}$ in the string models [1,2], and precisely this $\sigma$ has been used in the realistic potential model with linear confining potential $(\mathrm{CP})$ $[3,4]$. Also, systematization of radial excitations of light mesons, suggested in Ref. [5], has shown that their squared masses lie on linear, or approximately linear, radial trajectories in the $\left(M^{2}, n\right)$-plane $\left(n=n_{r}\right.$ is the radial quantum number) and has the slope, $\beta_{n}=(1.25 \pm 0.15) \mathrm{GeV}^{2}[5]$. Later in Refs. [6,7] a smaller slope $\beta_{n}=(1.143 \pm 0.013) \mathrm{GeV}^{2}$ was extracted from the Crystal Barrel data [8].

\footnotetext{
* Corresponding author.

b.l.g.bakker@vu.nl

badalian@itep.ru

Published by the American Physical Society under the terms of the Creative Commons Attribution 4.0 International license. Further distribution of this work must maintain attribution to the author(s) and the published article's title, journal citation, and DOI. Funded by SCOAP ${ }^{3}$.
}

It was also observed that the slopes of the $\left(M^{2}, J\right)$ trajectories for the masses with spin $S=0$ and $S=1$ differ only $\sim 10 \%$ [9] and it was assumed that within this accuracy a universal RT can exist in the $(l, n)$-plane,

$$
M^{2}(n, l)=a(l+n)+c
$$

with the universal slope $a=1.10(2) \mathrm{GeV}^{2}$ and the intercept $c=0.68 \mathrm{GeV}^{2}$. From Eq. (1) it follows that the masses of resonances with equal quantum number $N=$ $l+n$ have to be equal and this assumption agrees with the experimental masses of the vector resonances with $N=2$, 3, $4(S=1)$ (see Table I).

However, in another analysis of the experimental data, where the PDG masses and widths were used, a larger $\beta_{n}=(1.35 \pm 0.04) \mathrm{GeV}^{2}$ was extracted [11] and later, after reanalysis of the experimental data, the same authors have obtained a smaller $\beta_{n}=1.28(5) \mathrm{GeV}^{2}$ [12] with the

TABLE I. The PDG masses of the isovector resonances (in $\mathrm{MeV})[10]$ and $M(n, l)$ according to Eq. (1).

\begin{tabular}{lccc}
\hline \hline & $N=2$ & $N=3$ & $N=4$ \\
\hline Meson & $\rho_{3}(1690)$ & $\rho_{3}(1990)$ & $\rho_{3}(2250)$ \\
Mass & $1689(2)$ & $1982(14)$ & 2234 \\
Meson & $a_{2}(2 P)$ & $a_{4}(2040)$ & $\rho_{5}(2350)$ \\
Mass & $1705(40)$ & $1995(10)$ & $2330(35)$ \\
\hline \hline
\end{tabular}


conclusion that the universality of the radial and orbital RTs is not fulfilled at the level of 2.4 standard deviations. These results, irrespective of the fact whether slopes of radial and orbital RTs are equal or not, raise an important theoretical issue, namely, what dynamical effects are responsible for the values of the slopes, observed in experiments, and whether a universal RT exists or not. At present new studies of the RT nature continue [13,14].

A study of the light meson spectra in relativistic models shows that at first sight the RT parameters depend on the quark-antiquark potential $V_{0}(r)$ used, but, as shown in the relativistic string model [15-20], some additional corrections to the meson masses exist. The potential $V_{0}(r)$ was studied on a fundamental level in lattice QCD $[21,22]$ and the field correlator method [23] in the region $r \lesssim 1.2 \mathrm{fm}$. It was shown that in this region $V_{0}(r)$ is the sum of the linear CP $V_{\mathrm{C}}(r)=\sigma r$ and the gluon-exchange (GE) term: $V_{0}(r)=V_{\mathrm{C}}(r)+V_{\mathrm{GE}}(r)$. Precisely such a linear CP with string tension $\sigma=0.18 \mathrm{GeV}^{2}$, fixed by the slope of leading angular-momentum RT, was used in the relativistic models $[3,4]$, where a good description of the masses of low-lying states was obtained. However, to describe high excitations of light mesons, whose sizes $\geq 1.5 \mathrm{fm}$ are large, knowledge of the quark-antiquark potential at large distances is needed, which is not defined yet on a fundamental level, and in lattice QCD a flattening of the CP at $r \gtrsim 1.2 \mathrm{fm}$ is seen with large uncertainties. This flattening (screening) effect appears due to the creation of light $q \bar{q}$ holes (loops) in the Wilson loop and decreases the surface of the Wilson loop [17]. However, this effect was described only at a phenomenological level, assuming that a study of the high excitations can give important information about the $q \bar{q}$ interaction at large $r$ [15-17].

In light mesons one can use the universal GE potential, $V_{\mathrm{GE}}=-\frac{4 \alpha_{\mathrm{V}}(r)}{3 r}$, which is now well defined at small distances, since at present the QCD constant $\Lambda_{\overline{\mathrm{MS}}}$, as well as the vector constant $\Lambda_{\mathrm{V}}$, are known with a good accuracy for the number of flavours $n_{f}=3,4,5$ [24]. In particular, the value of $\Lambda_{\overline{\mathrm{MS}}}\left(n_{f}=3\right)=315(15) \mathrm{MeV}$ [24], or the corresponding $\Lambda_{\mathrm{V}}\left(n_{f}=3\right)=500(20) \mathrm{MeV}$ [25], appears to be larger than the value used in the past. However, the behavior of the strong coupling $\alpha_{\mathrm{V}}$ at small momenta and at large distances, as in the case of the CP, is still not determined [26] and it remains unclear whether a screening GE effect exists or not. This problem will be discussed in our paper.

In Refs. [15,17] it was shown that the main contribution to the light meson mass comes from the $\mathrm{CP}$ and as a first step it is instructive to consider the light meson spectrum, taking the purely linear $C P$ at all distances, and after that to take into account the flattening effect and other corrections. To make the theoretical analysis more clear we consider only isovector light mesons with $l \leq 3$ and pay special attention to calculations of the centroid masses. For that we use the relativistic string Hamiltonian (RSH), which describes the QCD string with spinless quarks at the ends and $m_{q}=0$ [18-20], while the spin-dependent interaction is taken as a perturbation; in this case the instantaneous $q \bar{q}$ potential reduces to the linear $C P$ plus the GE term.

The RSH is rather complicated and has different representations for large $l$ and small $l, l \leq 3$. Its basic term $H_{0}$, given by

$$
H_{0}=2 \sqrt{\boldsymbol{p}^{2}}+V_{0}(r), \quad(l \leq 3)
$$

is well known and widely used. Its eigenvalues (e.v.s) $M_{0}(n l)$ can be approximated by an analytical expression with great accuracy, if the light quark mass $m_{q}=0$. Notice that if in relativistic potential models the constituent quark mass, $\tilde{m}_{q} \sim(150-200) \mathrm{MeV}$, is used, then the parameters of the RTs depend on the value of the constituent quark mass.

The e.v.s $M_{0}(n l)$ provide the basic contributions to the meson mass and for the purely linear $C P$ the squared mass $M_{0}^{2}(n, l)$ can be approximated with great accuracy by the expression $[15,16]$,

$$
M_{0}^{2}(n, l)=\sigma(8 l+4 \pi n+3 \pi \xi(n l)),
$$

with $\xi(n l)=1.0$ with the exception of $\xi(1 S) \approx$ $\xi(1 P) \approx 1.05$. From the conventional representation of the RT as

$$
M^{2}(n, l)=\beta_{l} l+\beta_{n} n+\beta_{0},
$$

and using Eq. (3) for the purely linear $C P$, one obtains the following slopes and the intercept,

$$
\beta_{l}=8 \sigma, \quad \beta_{n}=4 \pi \sigma, \quad \beta_{0}=3 \pi \sigma .
$$

Now the following problem arises: if the conventional value of $\sigma=0.180 \mathrm{GeV}^{2}$ is taken, then all parameters of the RT (5) are significantly larger that those extracted from the experimental data $[5,6,11,12]$. Namely, the orbital slope $\beta_{l}=1.44 \mathrm{GeV}^{2}$ is $21 \%$ larger than $\beta_{l}(\exp )=1.13 \mathrm{GeV}^{2}$ of the leading RT. The radial slope $\beta_{n}=2.26 \mathrm{GeV}^{2}$ is about two times larger than the experimental $\beta_{n}(\exp ) \sim$ $1.2(1) \mathrm{GeV}^{2}$ for the states with $l \neq 0[5,6]$ (and 1.5 times larger than $\beta_{n}(l=0)$ for the radial $\rho$-trajectory), while the intercept $\beta_{0}=3 \pi \sigma=1.696 \mathrm{GeV}^{2}$ is 2.5 times larger than the corresponding one in Eq. (1). Notice that the value of the intercept cannot be decreased by introducing a negative (fitting) constant to the potential $V_{0}$, as it is often done in potential models. Moreover, appearance of this constant in the mass (or potential) violates the linearity of the RT. It is important that in the RSH, used here, the $q \bar{q}$ potential does not contain a fitting constant.

Our goal here is to understand what effects are responsible for the strong decrease of the intercept and the slopes of the RT (5), to establish the interrelation between the parameters of the RTs and the potential $V_{0}(r)$, and to show 
the role of the string and the self-energy corrections, which are present in the mass formulas. In contrast to our previous analysis $[15,16]$ we do not assume here that a screening of the GE potential $V_{\mathrm{GE}}$ takes place at distances $r<1.2 \mathrm{fm}$ and this assumption agrees with the results of Ref. [27], where it was shown that the screening effect of the GE potential is not seen at distances $r<1.0 \mathrm{fm}$. We also consider how the parameters of the RTs change for strong and weak vector coupling, taken in $V_{\mathrm{GE}}$.

Our analysis is restricted to orbital excitations with $l \leq 3$, because high orbital excitations with $l>3$ have to be considered in another approximation of the RSH, where the string corrections are very large and cannot be considered as a perturbation [20], and the ground state masses are described by the expression $M^{2}\left(l, n_{r}=0\right)=$ $2 \pi \sigma \sqrt{l(l+1)}$, in which the orbital slope of the leading RT $\beta_{l}\left(n_{r}=0\right)$ agrees well with the experimental number $2 \pi \sigma$, if $l \geq 3$.

We pay special attention to the negative correction produced by the self-energy (SE) term [28], whose magnitude remains large, $\delta_{\mathrm{SE}} \sim-300 \mathrm{MeV}$, even for high excitations of light mesons; being proportional to $1 / M(n l)$, it maintains linearity of the RT.

\section{THE MASS FORMULAS}

Here we present the structure of the mass formula, using the simplified version of the RSH, where the spindependent potentials, as well as the self-energy and the string contribution, are considered as a perturbation and the values of the angular momentum are restricted to $l \leq 3$ $[15,16]$. This RSH $H$ with $m_{q}=0$,

$$
H=\mu+\frac{\boldsymbol{p}^{2}}{\mu}+V_{0}(r)
$$

is expressed via the variable $\mu$, determined by the extremum condition, $\frac{\partial H}{\partial \mu}=0$. It gives $\mu=\sqrt{\boldsymbol{p}^{2}}$, i.e., $\mu$ is the kinetic energy of a quark. Then the Hamiltonian $H$ reduces to the form $H_{0}$ Eq. (2) and its e.v.s are defined by the spinless Salpeter equation (SSE),

$$
\left(2 \sqrt{\boldsymbol{p}^{2}}+V_{0}(r)\right) \varphi_{n l}(r)=M_{0}(n l) \varphi_{n l}(r) .
$$

The e.v. $M_{0}(n l)$ is an important part of the centroid mass $M_{\operatorname{cog}}(n l)$ and for an instantaneous $q \bar{q}$ interaction the potential $V_{0}(r)$ is taken as the sum of the confining and GE terms,

$$
V_{0}(r)=V_{\mathrm{C}}(r)+V_{\mathrm{GE}}(r),
$$

where the linear $\mathrm{CP} V_{\mathrm{C}}(r)$ is given by

$$
V_{\mathrm{C}}(r)=\sigma r, \quad \sigma=0.18 \mathrm{GeV}^{2},
$$

and also a flattened (screened) $\mathrm{CP}$ will be used,

$$
V_{\mathrm{f}}(r)=\sigma_{\mathrm{f}}(r) r
$$

The function $\sigma_{\mathrm{f}}(r)$ will be given in Sec. VI. The conventional form of $V_{\mathrm{GE}}(r)$ is

$$
V_{\mathrm{GE}}(r)=-\frac{4 \alpha_{\mathrm{V}}(r)}{3 r},
$$

if there is no a screening effect, and the problem of the GE screening will be discussed in Sec. V. The contributions from the GE potential to the masses of excited states are not large, $\lesssim 90 \mathrm{MeV}$, nevertheless, the GE correction is very important, decreasing all parameters of the RTs.

The masses can be calculated in two ways: either solving Eq. (7) with the potential $V_{0}(r)=V_{\mathrm{C}}(r)+V_{\mathrm{GE}}$, or considering $V_{\mathrm{GE}}(r)$ as a perturbation. It can be shown that for high excitations the exact and approximate values of mass coincide within $\sim 10 \mathrm{MeV}$. Then in the RSH the centroid mass $M_{\operatorname{cog}}(n l)$ includes the e.v. $M_{0}(n l)$ and three negative corrections: the self-energy and string corrections, and $\delta_{\mathrm{GE}}$,

$$
M_{\mathrm{cog}}(n l)=M_{0}(n l)+\delta_{\mathrm{GE}}+\delta_{\mathrm{str}}(n l)+\delta_{\mathrm{SE}}
$$

where the self-energy correction is the largest one and all three corrections together give a large negative contribution, $\sim-(400-500) \mathrm{MeV}$, while the e.v.s of the ground states $(n=0)$ are the following: $M_{0}(1 S)=1.339 \mathrm{GeV}$, $M_{0}(1 P)=1.792 \mathrm{GeV}, M_{0}(1 D)=2.155 \mathrm{GeV}$. It is worth to underline that the centroid mass $M_{\operatorname{cog}}(n l)$ does not contain a fitting negative constant $C_{0}$, usually introduced in potential models; this constant produces a nonlinear term $C_{0} M_{0}(n l)$ in the squared mass and violates the linearity of the RT. On the contrary, in our approach a negative contribution from the self-energy correction, $\delta_{\mathrm{SE}}(n l)$

$$
\delta_{\mathrm{SE}}(n l)=-\frac{\eta_{f} \sigma}{\mu(n l)}, \quad \eta(q \bar{q})=0.90
$$

is proportional to $1 / M_{0}$ via $\mu(n l)$ (see below) and therefore a nonlinear term does not appear in the RT. In Eq. (13) the number $\eta_{f}$ depends on the quark flavor and in light mesons we take $\eta_{f=q}=0.90$ [28]. The situation is different in heavy quarkonia, where the self-energy term is small and usually neglected, since, e.g., in bottomonium $\eta_{b} \sim 0.1$, $\mu_{b}(n l) \sim 5 \mathrm{GeV}$, and $\delta_{\mathrm{SE}} \sim-3 \mathrm{MeV}$. On the contrary, in a light meson $\delta_{\mathrm{SE}}$ has large magnitude, $\sim-(300-400) \mathrm{MeV}$, because the kinetic energy m.e. is small. It is important that this correction slightly decreases in higher excitations, but still remains large.

Another negative correction, the string correction $\delta_{\text {str }}(n l),(l=1,2,3)[15,16]$, given by 


$$
\delta_{\mathrm{str}}(n l)=-\frac{l(l+1) \sigma\left\langle r^{-1}\right\rangle}{8 \mu^{2}(n l)},
$$

increases for states with growing $l$ and decreases for larger $n$, however, its magnitude $\sim(-40,-80,-110)$ (in $\mathrm{MeV}$ ) for $l=1,2,3(n=0)$ is not large. Notice that the expression of $\delta_{\text {str }}$, Eq. (14) does not change if a flattened CP is taken, but in this case the string tension $\sigma$ has to be replaced by the averaged m.e. $\left\langle\sigma_{\mathrm{f}}(r)\right\rangle_{n l}$, which is different for every state and smaller than $\sigma$.

For high excitations with $l \neq 0$ knowledge of the centroid mass is very important, since due to their large sizes their fine-structure splittings are small and $M_{\text {cog }}(n l)$ practically coincides with the masses of the members of the multiplet. This does not refer to low $n S(1 P)$ states, where the spin-spin (fine-structure) splitting is not small. In particular, in the $n^{3} S_{1}$ states the hyperfine correction, equal to $-\frac{1}{4} \delta_{\mathrm{hf}}(n S)$, with

$\delta_{\mathrm{hf}}(n S)=\frac{8}{9} \alpha_{\mathrm{hf}} \tau(n S), \quad$ with $\quad \tau(n S)=\frac{\left|R_{n S}(0)\right|^{2}}{\mu^{2}(n S)}$,

is not small even for the $4^{3} S_{1}$ resonance. Calculations show that the ratio $\tau(n S)$, Eq. (15), weakly depends on the parameters of the GE potential, e.g., for the ground $1 S$ state $\tau(1 S)=(0.85-1.05) \mathrm{GeV}$ is obtained for different types of GE potentials. This fact allows us to extract $M_{\operatorname{cog}}(1 S)$ from experiment with an accuracy $\sim 10 \mathrm{MeV}$ (see below).

Notice that knowledge of $M_{\operatorname{cog}}(1 S)$ is of special importance since it determines the intercept of the leading $l$-trajectory $(n=0)$,

$$
M_{\mathrm{cog}}^{2}(l, n=0)=\beta_{l} l+\beta_{\mathrm{cog}}, \quad\left(n=n_{r}=0\right),
$$

with the intercept $\beta_{\operatorname{cog}}=M_{\operatorname{cog}}^{2}(1 S)$, where $M_{\operatorname{cog}}(1 S)=$ $M(\rho(1 S))-\frac{1}{4} \delta_{\text {hf }}(1 S)$. In Eq. (15) the hyperfine correction can be determined with $\sim 10 \mathrm{MeV}$ accuracy, if the universal hyperfine coupling $\alpha_{\mathrm{hf}}=0.33(1)$, the same as in heavylight mesons and bottomonium [29], and the theoretical number $\tau(1 S)=0.95(10) \mathrm{GeV}$ is used. It gives $\delta_{\mathrm{hf}}(1 S)=$ $280(25) \mathrm{MeV}$ and $M_{\operatorname{cog}}(1 S$, exp $)=\left(775-\frac{1}{4} 280(25)\right)=$ $705(6) \mathrm{MeV}$, so that the "experimental" intercept,

$\beta_{\operatorname{cog}}(\exp )=(0.705(6))^{2} \mathrm{GeV}^{2}=0.50(1) \mathrm{GeV}^{2}$,

is smaller than the intercept of the leading RT in the $\left(M^{2}, J\right)$-plane, defined by the mass of $\rho\left(1^{3} S_{1}\right): \beta_{0}(\exp )=$ $M^{2}(\rho(1 S, \exp ))=0.60 \mathrm{GeV}^{2}$.

Notice that for radial excitations the difference between the squared masses, $b_{n}^{2}=M_{\operatorname{cog}}^{2}(n+1, l)-M_{\operatorname{cog}}^{2}(n, l)$, of neighbouring states can depend on the radial quantum number $n$. If for all states with a given $l$ the numbers $b_{n}^{2}=b$ are equal, then the radial RT reduces to the radial RT, introduced in Ref. [5]:

$$
M(n, l)^{2}=M_{\mathrm{g}}^{2}+b n, \quad(l \text { fixed })
$$

where $M_{\mathrm{g}}(n=0, l)$ is the mass of the ground state.

\section{LINEAR CONFINING POTENTIAL}

The simplest way to show the structure of the RTs is to determine the light meson spectrum in a purely linear CP and consider other interactions as a perturbation; in this case the mass $M_{\operatorname{cog}}$ is defined by analytical expressions. Notice that the linear $\mathrm{CP}$ plays a special role in string theory as well as in the AdS approach [30]. In a linear potential the mass formula is simplified owing to the relations,

$M_{0}(n l)=4 \mu_{0}(n l), \quad \sigma\langle r\rangle_{n l}=2 \mu_{0}(n l)=1 / 2 M_{0}(n l)$.

In Table II we give the sizes $\left\langle\sqrt{r^{2}}\right\rangle_{n l}$, the m.e.s $\left\langle r^{-1}\right\rangle_{n l}$, and the e.v.s $M_{0}(n l)$, solving Eq. (7) with the linear potential $V_{\mathrm{C}}(r)$ with $\sigma=0.180 \mathrm{GeV}^{2}$.

Knowing the m.e.s $\left\langle r^{-1}\right\rangle_{n l}$ and the e.v.s $M_{0}(n l)$, we have observed that in a purely linear CP the m.e.s $\left\langle r^{-1}\right\rangle_{n l}$ can be approximated with an accuracy better than $2 \%$ as

$$
\begin{aligned}
\left\langle r^{-1}\right\rangle_{n l} & =M_{0}(n l) A(n l), \\
A(n l) & =\frac{0.262(l+2)}{(l+1)(l+n+2)}, \quad(l \neq 0) ; \\
\left\langle r^{-1}\right\rangle_{n} & =M_{0} A_{0}(n), \\
A_{0}(n) & =2 \frac{0.271}{n+2}=\frac{0.542}{n+2}, \quad(l=0),
\end{aligned}
$$

TABLE II. The eigenvalues $M_{0}(n l)$ (in GeV), the m.e.s $\left\langle\sqrt{r^{2}}\right\rangle_{n l}$ (in fm), $\left\langle r^{-1}\right\rangle_{n l}$ (in $\mathrm{GeV}$ ) of Eq. (7) with the linear potential $V_{c}(r)=\sigma r, \sigma=0.18 \mathrm{GeV}^{2}$.

\begin{tabular}{lccc}
\hline \hline State $(n+1) L$ & $M_{0}(n l)$ & $\left\langle\sqrt{r^{2}}\right\rangle_{n l}$ & $\left\langle r^{-1}\right\rangle_{n l}$ \\
\hline 1S & 1.339 & 0.82 & 0.364 \\
2S & 1.998 & 1.26 & 0.330 \\
3S & 2.498 & 1.58 & 0.296 \\
4S & 2.915 & 1.85 & 0.273 \\
1P & 1.792 & 1.06 & 0.236 \\
2P & 2.315 & 1.43 & 0.226 \\
3P & 2.750 & 1.72 & 0.214 \\
4P & 3.129 & 1.97 & 0.204 \\
1D & 2.155 & 1.24 & 0.187 \\
2D & 2.601 & 1.57 & 0.182 \\
3D & 2.990 & 1.84 & 0.176 \\
4D & 3.337 & 2.08 & 0.170 \\
1F & 2.465 & 1.41 & 0.159 \\
2F & 2.861 & 1.71 & 0.157 \\
3F & 3.215 & 1.96 & 0.153 \\
4F & 3.538 & 2.18 & 0.149 \\
\hline \hline
\end{tabular}


i.e., they are proportional to $M_{0}(n l)$. Then, with the use of the relations (19) and (20) all corrections to $M_{\operatorname{cog}}(n l)$ are given by analytical expressions.

For further analysis we rewrite the expression of $M_{0}(n l)^{2}$ (3) with $\sigma=0.180 \mathrm{GeV}^{2}$,

$M_{0}^{2}(n l)\left(\mathrm{in} \mathrm{GeV}^{2}\right)=(1.440 l+2.262 n+1.696 \xi(n l))$,

where the numbers $\xi(n l)=1.0$ with an accuracy better than $2 \%$ for all states, with the exception of $\xi(1 S)=1.057$ and $\xi(1 P)=1.045$. Note that in $M_{0}^{2}(n l)$ (21) the slopes $\beta_{l}=1.44 \mathrm{GeV}^{2}, \beta_{n}=2.26 \mathrm{GeV}^{2}$, and the intercept $\beta_{\operatorname{cog}}=$ $1.70 \xi \mathrm{GeV}^{2}$ are significantly larger than those, extracted from experimental data $[5,6,11,12]$, while due to the GE, the string, and the SE corrections the masses $M_{\operatorname{cog}}(n l)$ and the parameters of the RT decrease.

Then with the use of Eqs. (19) and (20) the orbital slope decreases owing to the string correction Eq. (14),

$\beta_{l}=\sigma\left(8-1.048 \frac{l(l+2)}{l+2+n}\right) \quad$ for $M=M_{0}+\delta_{\text {str }}$,

and in the general case it depends on the quantum number $l$ : $\beta_{l}(n=0)=1.251 \mathrm{GeV}^{2}$ for $l=1$ and $\beta_{l}(n=0)=$ $1.067 \mathrm{GeV}^{2}$ for $l=2$; for the radial RT with $n=1$ the slope $\beta_{l}(n=1)=1.299 \mathrm{GeV}^{2}$ for $l=1$ and $\beta_{l}(n=1)=$ $1.138 \mathrm{GeV}^{2}$ for $l=2$; for the daughter RT with $n=2$ $\beta_{l}(n=2)=1.327 \mathrm{GeV}^{2} \quad(l=1) \quad$ and $\quad \beta_{l}(n=2)=$ $1.188 \mathrm{GeV}^{2}$ for $l=2$. Thus with the string correction taken into account the orbital slope remains large and $l$-dependent, i.e., the RTs can be considered as approximately linear.

\section{A. The GE correction to the centroid mass}

Here we take the GE potential as a perturbation and later show that exact solutions of the SSE with $V_{0}(r)=$ $V_{\mathrm{C}}(r)+V_{\mathrm{GE}}$ give a contribution to the mass, which coincides with the GE correction with high accuracy (see Sec. VI). Using Eq. (20) the GE correction (11) can be rewritten as $\left(e_{\text {eff }}=\frac{4}{3} \alpha_{\text {eff }}\right)$

$$
\delta_{\mathrm{GE}}=-\frac{4}{3} \alpha_{\mathrm{eff}}(n l)\left\langle r^{-1}\right\rangle_{n l}=-e_{\mathrm{eff}} M_{0}(n l) A(n l),
$$

where in general the effective coupling, $\alpha_{\text {eff }}(n l)=\left\langle\alpha_{\mathrm{V}}(r)\right\rangle$ depends on the quantum numbers $n$ and $l$. However, in high excitations this dependence becomes weak because of their large sizes, $\gtrsim 1.4 \mathrm{fm}$, and the m.e.s $\alpha_{\text {eff }}(n l)$ are practically equal for all states, with the exception of the $1 S, 2 S$, and $1 P$ ground states, for which the asymptotic freedom (AF) behavior of the coupling is important (see below). For other states, the values of $\alpha_{\text {eff }}(n l)$ appear to be only $~ 3 \%$ smaller than the asymptotic coupling $\alpha_{\text {asym }}$. Therefore, for high excitations one can put $\alpha_{\text {eff }}(n l)=\alpha_{\text {asym. }}$. A typical $\alpha_{\text {asym }}$, used in relativistic models, lies in the range, 0.55-0.63 $[3,4,16]$. This value was also derived on a fundamental level $[23,25,26]$, where the uncertainty depends on the values of the vector QCD constant $\Lambda_{\mathrm{V}}\left(n_{f}=3\right)$ and the infrared (IR) regulator taken (see Sec. V). With the Coulomb constant $e_{\text {asym }}=\frac{4}{3} \alpha_{\text {asym }} \cong 0.72(4)$ and using the factor $A(n l)(20)$, one can see that

$$
\delta_{\mathrm{GE}}(n l)=-e_{\mathrm{asym}} M_{0}(n l) A(n l), \quad(l \neq 0, n \geq 1),
$$

is proportional to the e.v. $M_{0}(n l)$. It means that the GE correction gives a negative contribution to all parameters of the RT: the slopes $\beta_{l}, \beta_{n}$, and the intercept. Then the mass $M_{\mathrm{GE}}(n l)$ with the GE correction taken into account is

$$
\begin{aligned}
M_{\mathrm{GE}}(n l) & =M_{0}(n l) Z(n l), \quad \text { with } \\
Z(n l) & =\left(1-e_{\text {asym }} A(n l)\right), \quad(l \neq 0, n \geq 1) .
\end{aligned}
$$

but for the $n S$ states

$$
\begin{aligned}
M_{\mathrm{GE}}((n+1) S) & =M_{0}((n+1) S) Z_{0}(n), \\
Z_{0}(n) & =\left(1-e_{0}(n) A_{0}(n)\right),
\end{aligned}
$$

where $e_{0}(n) \neq e_{\text {asym }}$ and the quantities $A_{0}(n)$ are larger than $A(n l)$ with $l \neq 0$. From Eq. (25) one can see that the parameters of the RTs can depend on the quantum numbers through the factor $A(n l)$, but in high excitations this dependence is weak because the term $e_{\text {asym }} A(n l)$ is small even for a strong GE potential. We choose the Coulomb constant, $e_{\text {asym }}=0.76$ (or $\alpha_{\text {asym }}=0.57$ ) (see below) and define the average $\langle A(l$, fixed $n)\rangle=$ $\frac{1}{2}(A(l=1, n)+A(l=2, n))$. Then for $n=0$ one finds $e_{\text {asym }}\langle A(l, n=0)\rangle=0.084(16), Z(l, n=0)=0.916(16)$, and $Z^{2}(l, n=0)=0.839(30)$. For $n=1$ with $e_{\text {asym }}\langle A(l, n=1)\rangle=0.064(11)$, the factor $Z(n=1)=$ $0.936(11), Z^{2}(n=1)=0.876(20)$ is larger; for the daughter RT with $n=2,\langle A(n=2)\rangle=0.052(8), Z^{2}(n=2)=$ $0.899(14)$ are obtained. We can conclude that in the linear $\mathrm{CP}$ due to the factor $Z^{2}(n l)$, defined by the GE correction, the orbital slope decreases by $\sim(10-16) \%$, but still remains large, $\beta_{l} \sim(1.24-1.30) \mathrm{GeV}^{2}$. Also the intercept decreases, although its value, $\left.3 \pi \sigma Z^{2}(n) \sim(1.43-1.53)\right) \mathrm{GeV}^{2}$, remains large for all RTs.

The GE corrections give a contribution to the kinetic energy m.e.s, denoted as $\mu_{\mathrm{GE}}$, see Eq. (27), which are given in Table III together with the SE and the string corrections, and $M_{\operatorname{cog}}(n l)$. From this table one can see that for the ground states their masses agree with experiment, while for the $2 S, 2 P$, and $2 D$ states and higher excitations the masses $M_{\text {cog }}(n l)$ are larger by (100-200) $\mathrm{MeV}$ than the experimental values and the only way to decrease these masses is to take into account a flattened, or screened, CP. Note that without the SE and string corrections the masses 
$M_{\mathrm{GE}}(n l)=M_{0}+\delta_{\mathrm{GE}}$ are larger by $\sim(300-400) \mathrm{MeV}$ than the experimental masses $M_{\operatorname{cog}}$ (exp).

There exists another effect, produced by the GE potential, which increases the quark kinetic energy and for the states with $l \neq 0$ this m.e. can be approximated (with an accuracy better than $5 \%$ ) by

$$
\begin{aligned}
\mu_{\mathrm{GE}}(n l) & =\mu_{0}(n l)+\frac{1}{4} \delta_{\mathrm{GE}}(n l) \\
& =\frac{M_{0}(n l)}{4}\left(1+e_{\mathrm{eff}}(n l) A(n l)\right) .
\end{aligned}
$$

The kinetic energy $\mu_{\mathrm{GE}}$ is larger than $\mu_{0}(n l)$ and has to be taken into account in the self-energy and the string corrections, which decrease due to this effect. In some cases, instead of the approximation (27), one can use another approximation for $\mu_{\mathrm{GE}}$,

$$
\begin{aligned}
\mu_{\mathrm{GE}}(n l) & =1.11 \mu_{0}(n l)=0.275 M_{0}(n l), \\
\mu_{\mathrm{GE}}(n=l=0) & =1.21 \mu_{0}(l=n=0)=0.3025 M_{0}(l=n=0) .
\end{aligned}
$$

\section{B. The string correction}

With the use of the modified kinetic energy $\mu_{\mathrm{GE}}(28)$ the string correction, proportional to $\left\langle r^{-1}\right\rangle_{n l}=A(n l) M_{0}(n l)$ (13), can be written as

$$
\delta_{\mathrm{str}}(n l)=-l(l+1) \frac{\sigma\left\langle r^{-1}\right\rangle}{8 \mu_{\mathrm{GE}}^{2}}=-l(l+1) 1.623 \sigma A(n l) M_{0}^{-1},
$$

i.e., it is proportional to $l$ and contributes only to the orbital slope $\beta_{l}$, decreasing its value. Since the string correction is not large $\left(\delta_{\text {str }} \sim-45 \mathrm{MeV}\right.$ for the $1 P$ state, $\sim-80 \mathrm{MeV}$, $\sim-105 \mathrm{MeV}$, respectively, for the $1 D$ and $1 F$ ground states, and smaller for radial excitations (see Table III), it decreases the orbital slope only by $(5-10) \%$. Nevertheless, taking into account the string correction improves the agreement of the theoretical $\beta_{l}$ with the experimental value $\beta_{l}(\exp )=1.13 \mathrm{GeV}^{2}[11]$.

\section{The self-energy correction}

The SE correction (13) is of special importance in light mesons and with the modified kinetic energy Eq. (27) can be rewritten as

$$
\delta_{\mathrm{SE}}(n l)=-\frac{0.9 \sigma}{\mu_{\mathrm{GE}}}=-\frac{3.243 \sigma}{M_{0}(n l)},
$$

being proportional to $M_{0}^{-1}(n l)$. Therefore, $\delta_{\mathrm{SE}}$ produces a negative constant in the squared mass and strongly decreases the intercept, but does not change the radial and orbital slopes.

In Table III the centroid mass $M_{\operatorname{cog}}(n l)(10)$, the corrections $\delta_{\mathrm{GE}}, \delta_{\mathrm{str}}, \delta_{\mathrm{SE}}$, defined by the Eqs. (24), (29), and (30), are given together with the averaged kinetic energy $\mu_{\mathrm{GE}}(27)$ and the experimental values of $M_{\operatorname{cog}}(n l)$, which are known, if the experimental masses of all members of a multiplet are measured. In the cases where in the PDG [10] only the mass of the highest state with $J=l+1$ is given, then an inequality $M_{\operatorname{cog}}(n l$, exp $)<M(J=l+1$, exp) takes place. For illustration we have chosen the vector coupling equal to a constant, $\alpha_{\mathrm{V}}=0.482$, or $e=0.643$, and neglected the asymptotic freedom (AF) effect.

In a more realistic case one can take $\alpha_{\text {eff }}(n l)=\alpha_{(\text {asym) }}$, i.e., $e_{\text {eff }}(n l)=e_{\text {asym }}$, for all states (with exception of the states $1 S, 2 S$, and $1 P)$; then $\left.Z(n l)=1-e_{\text {asym }} A(n l)\right)$ and the expression of the centroid mass $M_{\operatorname{cog}}(n l)$ is simplified to

$$
\begin{aligned}
M_{\operatorname{cog}}(n l)= & M_{0}(n l) Z(n l)-1.623 l(l+1) \sigma \frac{A(n l)}{M_{0}}-\frac{3.24 \sigma}{M_{0}}, \\
& (l \neq 0, n \geq 1) .
\end{aligned}
$$

TABLE III. The centroid mass $M_{\mathrm{cog}}(n l)(12)$, the kinetic energy $\mu_{\mathrm{GE}}(n l)$, the GE correction $\delta_{\mathrm{GE}}$ with $e=0.643$ (in $\mathrm{GeV}$ ), and the corrections $\delta_{\mathrm{str}}, \delta_{\mathrm{SE}}($ in $\mathrm{GeV}$ ) in the purely linear confining potential.

\begin{tabular}{lcccccc}
\hline \hline State $(n+1) L$ & $\delta_{\mathrm{GE}}$ & $\mu_{\mathrm{GE}}$ & $\delta_{\mathrm{SE}}$ & $\delta_{\text {str }}$ & $M_{\mathrm{cog}}(n l)$ & $M_{\operatorname{cog}}, \exp [10]$ \\
\hline IS & -0.234 & 0.405 & -0.400 & 0 & 0.705 & $0.705(6)$ \\
2S & -0.212 & 0.553 & -0.293 & 0 & 1.493 & $1.424(25)$ \\
3S & -0.182 & 0.672 & -0.241 & 0 & 2.067 & $1.875(5)$ \\
4S & -0.168 & 0.773 & -0.210 & 0 & 2.529 & Absent \\
1P & -0.152 & 0.486 & -0.333 & -0.044 & 1.263 & $<1.318$ \\
2P & -0.146 & 0.615 & -0.263 & -0.027 & 1.879 & $<1.732(9)$ \\
3P & -0.138 & 0.722 & -0.224 & -0.018 & 2.369 & Absent \\
1D & -0.120 & 0.569 & -0.285 & -0.076 & 1.674 & $\approx 1.69$ \\
2D & -0.117 & 0.679 & -0.238 & -0.052 & 2.194 & $\approx 1.990$ \\
3D & -0.113 & 0.776 & -0.209 & -0.038 & 2.630 & absent \\
1F & -0.102 & 0.642 & -0.252 & -0.102 & 2.009 & $\sim 1.995(10)$ \\
2F & -0.101 & 0.741 & -0.219 & -0.076 & 2.465 & Absent \\
\hline \hline
\end{tabular}


Then the squared mass has a clear structure,

$$
\begin{aligned}
M_{\mathrm{cog}}^{2}(n l)= & M_{0}^{2} Z^{2}(n l)-3.246 \sigma l(l+1) A(n l) Z(n l) \\
& -6.48 \sigma Z(n l)+\delta_{\mathrm{SE}}^{2}+\text { small terms. }
\end{aligned}
$$

From Eq. (32) several conclusions can be drawn. One can see that the corresponding RT is nonlinear through the terms $A(n l)$ and $Z(n l)$, however, taking the averaged $\bar{A}=$ $\langle A(n)\rangle$ for a given $l$, the radial RTs can be considered as approximately linear.

In the radial slope the GE correction ( $l$ is fixed) is defined by $Z(l)^{2}$,

$$
\beta_{n}(\text { fixed } l)=4 \pi \sigma Z^{2}(l)=4 \pi \sigma\left(1-e_{\text {eff }} \bar{A}(l)\right)^{2},
$$

and the value $\beta_{n}(l)=(1.96-2.06) \mathrm{GeV}^{2}$ remains large for any $l$, being $\sim 70 \%$ larger than the experimental radial slope, $\beta_{n} \sim 1.2(1) \mathrm{GeV}^{2}(l \neq 0)$ [5-7], even if the strong GE potential is used. Just owing to the large radial slope the large masses $M_{\operatorname{cog}}(n l)$, given in Table III, are obtained.

In that table we give $M_{\operatorname{cog}}(n l)$, calculated with $e_{\text {eff }}=0.643$, which is smaller than $e_{\text {asym }}$, and in this case $M_{\text {cog }}(2 S)$ is $\sim 70 \mathrm{MeV}$ larger than $M\left(\rho(2 S) ; M_{\text {cog }}(2 P)\right.$ is larger by $\sim 150 \mathrm{MeV}$ than $M(a(1320))$, and $M_{\operatorname{cog}}(2 D)$ is larger than $\rho_{3}(1990)$ by $\sim 200 \mathrm{MeV}$. These masses would be only $\sim 30 \mathrm{MeV}$ smaller, if the larger $e_{\text {eff }}=e_{\text {asym }}=0.76$ was used.

The orbital slope decreases owing to both the GE and the string corrections and with $Z(n l)=1-e_{\text {asym }} A(n l)$,

$$
\beta_{l}=\sigma\left(8 Z^{2}(n l)-0.851 \frac{l+2}{l+2+n} Z(n l)\right)
$$

where for large $l$ and $n=0,1$ the contribution from $\delta_{\mathrm{str}}^{2}$, which was neglected in Eq. (34), may be not small.

For the leading Regge trajectory (LRT) with $n=0$ the orbital slope with $\bar{A}(l)=0.084(16)$ and $e_{\text {asym }}=0.76$, is $\beta_{l}(n=0)=5.933 \sigma=1.07(3) \mathrm{GeV}^{2}$, which is in good agreement with the experimental value $\beta_{l}(\exp )=$ 1.13(1) $\mathrm{GeV}^{2}[7,11]$. However, in a daughter RT, e.g., with $n=2\left[\bar{A}(l)=0.052(21), e_{\text {asym }} \bar{A}(l)=0.044(16)\right]$ the orbital slope $\beta_{l}(n=2)=6.77 \sigma=1.22 \mathrm{GeV}^{2}$ is $14 \%$ larger than $\beta_{l}(n=0)$ and this RT is not parallel to the LRT.

From Eq. (32) one can see that the contributions to the intercept come from the GE and self-energy corrections. For the LRT the intercept $\beta_{\operatorname{cog}}(n=0)=0.50(1) \mathrm{GeV}^{2}$ was already determined from the experimental value of $M(\rho(1 S)$, while in the orbital RT with $(n=1)$ $(\bar{A}(n=1)=0.058(14), \quad e A=0.044(11), \quad Z(n=1)=$ $0.956)$ a cancellation of two terms occurs,

$\beta_{\mathrm{cog}}=\sigma\left(3 \pi 0.956^{2}-6.195\right)=2.42(18) \sigma=0.44(3) \mathrm{GeV}^{2}$, and the calculated intercept agrees with the experimental intercept, $\beta_{\operatorname{cog}}(\exp )=0.50(1) \mathrm{GeV}^{2}$, within the accuracy of the calculations.

Thus we conclude that in the purely linear CP with all corrections taken into account and large Coulomb constant, $e \sim(0.64-0.76)$, the masses of the ground states agree with experiment, while the masses of first excitations exceed the experimental values by $\sim(100-150) \mathrm{MeV}$.

\section{THE LEADING REGGE TRAJECTORY}

The leading RT describes the ground states with $S=1$, where the $1 S, 1 P$, and $1 D$ states have relatively small sizes (see Table II), so for them the use of the linear CP can be justified. In the $\left(J, M^{2}\right)$-plane, $(J=l+1)$, the LRT can be written as $M^{2}(J, n=0)=(1.13 J-0.53) \mathrm{GeV}^{2}$, or in the $\left(l, M^{2}\right)$-plane it can be rewritten similar to that for $M_{\mathrm{cog}}^{2}(n l)(16)$,

$$
\begin{aligned}
& M^{2}(J=S+1, n=0)=\beta_{l} l+\beta_{0 J}, \quad \text { with } \\
& \beta_{J 0}=M^{2}(\rho(1 S))=0.60 \mathrm{GeV}^{2},
\end{aligned}
$$

where the intercept $\beta_{0 J}$ is larger than the intercept $\beta_{\operatorname{cog}}=$ $M_{\operatorname{cog}}(1 S)^{2}=0.705(6)^{2} \mathrm{GeV}^{2}=0.50(1) \mathrm{GeV}^{2}$ (17).

To determine the intercept of the LRT it is not sufficient to take into account the self-energy correction, otherwise in a purely linear $\mathrm{CP}$ (with $M_{0}(1 S)=1.339 \mathrm{GeV}$ and $\left.\mu_{0}(1 S)=\frac{M_{0}}{4}=0.335 \mathrm{MeV}\right)$ one would obtain the mass $M_{\operatorname{cog}}(1 S, \operatorname{lin})=M_{0}(1 S)-\frac{3.6 \sigma}{M_{0}(1 S)}=0.826 \mathrm{GeV}$

$\left(\sigma=0.180 \mathrm{GeV}^{2}\right)$, which is even larger than the experimental mass of the $\rho(1 S)$ meson. As was shown in previous section, owing to the GE potential, the kinetic energy increases from the value $\mu_{0}(1 S)=0.335 \mathrm{GeV}$ to $\mu_{\mathrm{GE}}(1 S)=(0.395 \div 0.415) \mathrm{GeV}=0.405(10) \mathrm{GeV}$, where the uncertainty depends on the uncertainty in the QCD vector constant $\Lambda_{\mathrm{V}}\left(n_{f}=3\right.$ ) taken (see Table IV and Sec. VI), and the value of $\mu_{\mathrm{GE}}=0.405(10) \mathrm{GeV}$ is obtained from the exact solutions of the SSE (7).

With $\mu_{\mathrm{GE}}(1 S)=0.405(10) \mathrm{GeV}$ the self-energy correction, $\delta_{\mathrm{SE}}(1 S)=-0.400(10) \mathrm{GeV}$, decreases, being $\sim 100 \mathrm{MeV}$ smaller than that for $\mu_{0}(1 S)$.

In the LRT the effective constants $\alpha_{\text {eff }}(1 S)=\left\langle\alpha_{\mathrm{V}}(r)\right\rangle_{1 S}$ are not equal for all states, since the AF effect decreases $\alpha_{\text {eff }}$ for the $1 S$ and $1 P$ states by $\sim(10-15) \%$, while for the states with $l=2,3$ their couplings are practically equal to the asymptotic coupling $\alpha_{\text {asym }}$.

It is of interest to notice that the coupling $\alpha_{\text {eff }}(1 S)$ can be extracted from experiment, if one uses the "experimental" value of the centroid mass, $M_{\operatorname{cog}}(1 S$, exp $)=0.705(6) \mathrm{GeV}$ (17). Taking $\mu_{\mathrm{GE}}=0.405(10) \mathrm{GeV}, \delta_{\mathrm{SE}}(1 S)=0.400 \mathrm{GeV}$, and the mass $M_{\operatorname{cog}}(1 S)$ given by 
TABLE IV. The averaged values of the orbital and the radial slopes, and the intercept (in $\mathrm{GeV}^{2}$ ) of the Regge trajectories for the linear CP $V_{C}(r)\left(\sigma=0.18 \mathrm{GeV}^{2}\right)$ and different gluon-exchange terms $V_{\mathrm{GE}}$.

\begin{tabular}{lccc}
\hline \hline & Linear CP & Linear CP + weak $V_{\mathrm{GE}}$ & Linear CP + strong $V_{\mathrm{GE}}$ \\
Potential Corrections & 0 & $\delta_{\mathrm{SE}} \neq 0$, & 0.57 \\
\hline$\alpha(\mathrm{eff})$ & 0 & 0.30 & $1.13(2)$ \\
$\left\langle\beta_{l}(n=2)\right\rangle$ & 1.440 & $1.225(1)$ & $1.13(3)$ \\
$\left\langle\beta_{l}(n=1)\right\rangle$ & 1.440 & $1.17(4)$ & $1.09(2)$ \\
$\left\langle\beta_{l}(n=0)\right\rangle, l \neq 0$ & 1.440 & $1.12(3)$ & $1.97(5)$ \\
$\left\langle\beta_{n}\right\rangle(l \neq 0)$ & 2.262 & $2.14(2)$ & $0.46(2)$ \\
$\left\langle\beta_{\mathrm{cog}}\right\rangle,(n=2)$ & 1.696 & $0.47(1)$ & $\delta_{\mathrm{SE}}$ \\
\hline \hline
\end{tabular}

$$
\begin{aligned}
M_{\mathrm{cog}}(1 S) & =M_{0}(1 S)+\delta_{\mathrm{SE}}(1 S)+\delta_{\mathrm{GE}}(1 S) \\
& =\left(1.339-0.400(10)-e_{0}(1 S)\left\langle r^{-1}\right\rangle_{1 S}\right) \mathrm{GeV} \\
& =0.705(6) \mathrm{GeV}
\end{aligned}
$$

one determines the effective constant $e_{0}(1 S)=0.643(41)$ (here $\left\langle r^{-1}\right\rangle_{1 S}=0.364 \mathrm{GeV}$ ), or the effective coupling, $\alpha_{\text {eff }}(1 S)=0.482(31)$, with a theoretical error $\sim 6 \%$. Note that the lower limit of $\alpha_{\mathrm{eff}}(1 S)=0.45$, which appears to be significantly larger than $\alpha_{\mathrm{V}}=0.30$, used in our paper before [15]. At the same time the upper limit of this coupling, equal to 0.51 , is smaller than $\alpha_{\text {asym }}=0.57(3)$, $e_{\text {lasym }}=0.76$, used in high excitations, confirming the influence of the AF effect.

For the ground $1 S$ state from Eq. (20) one has $A_{0}(n=0)=0.272$ and with the fitted value $e_{0}(1 S)=$ $0.643(41)\left(\delta_{\mathrm{GE}}(1 S)=-0.234(15) \mathrm{GeV}\right)$, one obtains the factor $e_{0}(1 S) A_{0}(n=0)=0.175(11)$, or

$$
Z_{0}(1 S)=1-e_{0} A_{0}(n=0)=0.825(16)
$$

i.e., the factor $Z_{0}^{2}(1 S)$ decreases the squared mass (37) by $\sim 32 \%$ and provides the correct value of the intercept, $\beta_{\operatorname{cog}}(1 S)=\beta_{\operatorname{cog}}(\exp )=0.50(1) \mathrm{GeV}^{2}$. In the ground states with $l \neq 0$ the GE correction (24), proportional to $M_{0}(l, n=0)$,

$\delta_{\mathrm{GE}}(1 l)=-0.262 e_{l}(n=0) \frac{M_{0}(1 l)}{l+1}, \quad(l \neq 0, n=0)$,

in general contains different values of $e_{l}(n=0)$ and $Z(l, n=0)$

$$
Z(l, n=0)=1-0.262 \frac{e_{l}(n=0)}{l+1}, \quad(l \neq 0) .
$$

Then the centroid mass can be rewritten as,

$$
M_{\mathrm{cog}}(1 l)=M_{0}(1 l) Z(l, 0)-\frac{3.243 \sigma}{M_{0}(1 l)}-\sigma l \frac{0.427}{M_{0}}, \quad(l \neq 0) .
$$

If the approximate relation $\mu_{\mathrm{GE}}=1.11 \mu_{0}$, following from Eqs. (27) and (28), is used, then the squared mass $M_{\mathrm{cog}}^{2}(1 l)$ is

$$
\begin{aligned}
M_{\mathrm{cog}}^{2}(1 l)= & M_{0}^{2} Z^{2}(l, 0)-0.851 \sigma l Z(l, 0)-6.486 \sigma Z(l, 0) \\
& +\delta_{\mathrm{SE}}^{2}+\text { small terms }
\end{aligned}
$$

Here, in the orbital slope the constants $e_{l}(n=0)$ are different for the $1 P$ and the ground states with $l \geq 2$, for which the asymptotic value, $e_{l}(l, n=0)=e_{\text {lasym }}$ can be used, while due to the AF effect the coupling $\alpha_{\text {eff }}(1 P)$ has a value close to that for the $1 S$ state. Here we take $e_{l}(l=1, n=0)=e_{0}=0.643(41)$ and $e_{l}(l \geq 2, n=0)=$ $e_{\text {asym }}=0.76$. Then with $A(l=1, n=0)=0.131$, $A(l=2, n=0)=0.0873$ and the average, $\left\langle e_{l} A(l, n=0)\right\rangle=$ $0.075(5)$, and $Z(n=0)=0.925(5)$ from the Eq. (42) the orbital slope is

$$
\begin{aligned}
\beta_{l}(n=0) & =\sigma\left(\left(8 Z^{2}(n=0)-0.851 Z(n=0)\right)\right. \\
& =6.06(7) \sigma=1.09(1) \mathrm{GeV}^{2},
\end{aligned}
$$

which agrees almost precisely with experimental slope, $\beta_{l}=1.13(1)$ [7].

Also with the chosen constants $e_{l}(1 P)=0.643(41)$ and $Z(1 P)=0.916(5)$ the intercept, $\beta_{\operatorname{cog}}(1 P)=\sigma\left(3 \pi Z(n=0)^{2}-\right.$ $6.484 Z(n=0))+\delta_{\mathrm{SE}}^{2}=2.58(5) \sigma=0.46(1) \mathrm{GeV}^{2}$, is obtained in good agreement with the experimental number, $\beta_{\mathrm{cog}}=0.50(1)$, where a contribution from the squared correction $\delta_{\mathrm{SE}}^{2}=0.11 \mathrm{GeV}^{2}$ is $\sim 25 \%$.

In Table III for simplicity we give the centroid masses with equal Coulomb constant, $e_{l}=e_{0}=0.643$, and the masses $M_{\operatorname{cog}}(1 P)=1263 \mathrm{MeV}, M_{\operatorname{cog}}(1 D)=1674 \mathrm{MeV}$, $M_{\operatorname{cog}}(1 F)=2009 \mathrm{MeV}$ (without fine-structure splitting) turn out to be in good agreement with the experimental masses of $a_{2}(1320), \rho_{3}(1690)$, and $a_{4}(2040)$ (its mass, $\left.\left.M(\exp )=1995_{-8}^{+10} \mathrm{MeV}\right)[10]\right)$. This agreement indicates that in the ground states with $l \geq 2$ the fine-structure splittings are not large. Notice that the magnitudes of the string corrections, which are equal to $-48 \mathrm{MeV}$, $-81 \mathrm{MeV}$, and $-142 \mathrm{MeV}$, for $l=1,2,3$, respectively, grow for increasing $l$. 
In conclusion in Table IV we give the parameters of the RTs for different types of the potential $V_{0}(r)$ : for the purely linear $\mathrm{CP}$, the linear $\mathrm{CP}+$ weak $V_{\mathrm{GE}}$, and for the linear $\mathrm{CP}+$ strong $V_{\mathrm{GE}}$.

Thus, our analysis of the RTs, when the CP is linear at all distances and the SE, the string and the GE contributions are taken as a perturbations, has allowed to get analytical expressions for the masses and the parameters of the RTs, which have several characteristic features:

(1) The radial slope, $\beta_{n} \cong 2.0 \mathrm{GeV}^{2}(l=1,2,3)$, remains larger than $\beta_{n}(\exp )=1.2(1) \mathrm{GeV}^{2}$ by $\sim 60 \%$, irrespective of the strength of the GE potential used, and the cases with $\alpha_{\text {eff }}=0.30$ and $\alpha_{\text {eff }}=0.57$ were compared.

(2) On the contrary, the orbital slope of the LRT $\beta_{l}(n=0)=1.09(1) \mathrm{GeV}^{2}$ agrees with the experimental value, if the strong vector coupling $\alpha_{\mathrm{V}} \sim$ $0.53(4)$ is taken. This choice of the coupling is preferable, since for small coupling, $\alpha_{\mathrm{V}}=0.30$, the orbital slope $\beta_{l}(n=0) \sim 1.20(2) \mathrm{GeV}^{2}$, and the mass of $\rho(1 S)$ is larger than in experiment.

(3) In the linear CP the orbital slope of the daughter RTs $(n \geq 1)$ is $(10-15) \%$ larger than $\beta_{l}(n=0) \approx$ $\beta_{l}(\exp )=1.13(1) \mathrm{GeV}^{2}$, even if the strong GE potential is used. Precisely for that reason the $q \bar{q}$ interaction has to be modified at large distances.

(4) The largest effect from the GE potential refers to the masses of the $n S$ states, which increases the radial slope of the $\rho(n S)$-trajectory (see Sec. VI).

\section{THE GLUON-EXCHANGE POTENTIAL AT LARGE DISTANCES}

Here we use the conventional $q \bar{q}$ potential $V_{0}(r)$ as a simple sum, Eq. (8). This representation is confirmed by the Casimir scaling effect, observed in lattice QCD [31] and derived in the field correlator method [32]. Meanwhile, this choice as the sum of two terms does not imply that each term, the $\mathrm{CP}$ and the GE potentials, is described by the simple expression as in Eqs. (9) and (11) at all distances. Moreover, in lattice QCD the linear behavior of the CP is proved to be valid only in the region $r \lesssim 1.2 \mathrm{fm}$, while for $r>1.2 \mathrm{fm}$, the flattening, or screening, of the $\mathrm{CP}$ is seen, but the details of $V_{\mathrm{GE}}$ are not studied yet and the flattened $\mathrm{CP}$ was only introduced phenomenologically in several models [15,33,34].

Also the expression of the GE potential (11), taken from perturbative QCD, in a strict sense is valid only up to the momentum $q^{2} \gtrsim 1.5 \mathrm{GeV}^{2}$ in momentum space, or down to very small distances, $r<0.1 \mathrm{fm}$ in coordinate space [35,36]. Therefore, to use $V_{\mathrm{GE}}(r)$ in coordinate space in the whole region, one must first regularize the vector coupling $\alpha_{\mathrm{V}}\left(q^{2}\right)$ in momentum space and then regularize $\alpha_{\mathrm{V}}(r)$ by using in Eq. (47) (see below) the regularized $\alpha_{\mathrm{V}}\left(q^{2}\right)$. Notice that the asymptotic values of $\alpha_{\mathrm{V}}$ are equal in momentum and coordinate space [36]. It seemingly supports the idea that the OGE and confining interactions are not independent.

Here we follow the detailed analysis from Ref. [27], which reveals at least three important effects, which can modify $V_{\mathrm{C}}(r)$ and $V_{\mathrm{GE}}$ owing to background fields.

(1) The gauge invariance of the gluon exchange in the confining background requires the propagating gluon to be inside the confining film (the surface, filled by the background fields), connecting the $q$ and $\bar{q}$ trajectories. The resulting area of the film should obey the Wilson minimal-area law [27].

(2) The propagating gluon can create $g g$ (gluon-gluon) loops in the confining film only in the higher $O\left(\alpha_{s}\right)$ orders, which introduces a new mass parameter $M_{\mathrm{B}}$ [37], expressed via the string tension, and its value, $M_{\mathrm{B}}^{2}=2 \pi \sigma$, defined with $10 \%$ accuracy, enters in the evolution equation together with $q^{2}$, making the coupling dependent on the variable $\left(q^{2}+M_{\mathrm{B}}^{2}\right)[16,37]$.

(3) If the confining string is long, it can create light $q \bar{q}$ holes in the confining film, thus decreasing the surface of the Wilson loop (i.e., the film surface). A finite density of these holes gives rise to the flattening of the confining potential at large $r$ and due to the flattening effect the masses of high excitations decrease, since their effective string tension is smaller for large $r$ than that in the region $r<1.2 \mathrm{fm}[15,16]$.

The points 2 and 3 were discussed in the literature, while the properties of the GE interaction needs some comments and the behavior of $V_{\mathrm{GE}}$ at large distances, called the color Coulomb screening effect, was studied first in Ref. [38] and recently in Ref. [27].

In the simplest treatment [38] a deformation of the confining film, owing to propagating the quark and the antiquark trajectories, was not optimal and due to the transformation of a gluon into a one-gluon glue lump [23], the screening of the GE interaction, $V_{\mathrm{OGE}}=-\frac{4 \alpha_{\mathrm{v}}}{3 r} f_{\mathrm{scr}}$, was shown to exist already at distances $\sim 0.6 \mathrm{fm}$. Notice that such a strong screening is not seen in bottomonium, where $\chi_{b}(2 P)$ and $\Upsilon(3 S)$ have sizes $\sim 0.6 \mathrm{fm}$ and $0.7 \mathrm{fm}[25,39]$.

In a more accurate treatment [27] one has to maintain full gauge invariance of the OGE interaction, and in addition take into account the Wilson criterium of the minimal area law of the resulting film surface, which contains both OGE and confinement. Denoting the time distances between consecutive gluon-exchanges as $L$, at large $L$, one can consider this system as a hybrid excitation of the $q \bar{q}$ system of size $L$. Then, the mass of a transverse excitation is $m_{\mathrm{scr}} \cong$ $\frac{\sqrt{12}}{L}$ [40]. On the other hand, the average value of $L$ enters into the action's (the total Lagrangian) exponent as $\exp \left(-V_{\mathrm{OGE}} L\right) \sim \mathcal{O}(1)$, or $L^{-1} \approx \frac{4 \alpha_{V}}{3 r}$. As a result one obtains an estimate of the screening mass (if $\alpha_{\mathrm{V}} \cong 0.50$ ) [27], 


$$
m_{\mathrm{scr}} \approx \sqrt{12} \frac{4 \alpha_{V}}{3 r_{\mathrm{eff}}} \lesssim 0.40 \mathrm{GeV} \quad\left(r_{\mathrm{eff}} \gtrsim 1 \mathrm{fm}\right) .
$$

Note that this estimate refers to large distances, $r \gtrsim 1 \mathrm{fm}$, where the deformation of the surface, due to the gluon exchange, is significant, while the screening (and deformation) is suppressed for the smaller $L$.

We may conclude that the flattening of the $\mathrm{CP}$ and the screening of $V_{\mathrm{GE}}$ start at approximately the same distances, $r>(1.0-1.2) \mathrm{fm}$, but their nature is different. The flattening effect appears due to creation of light $q \bar{q}$ holes, which, decreasing the film surface (or the Wilson loop), also decreases the string tension at large distances.

The screening of the GE potential occurs because the movement of the gluon is restricted inside the film surface and gives rise to a deformation of the film, so that due to confinement a kind of "gluon mass," $m_{\text {scr }} \sim 0.4 \mathrm{GeV}$, appears. Thus the analysis of Ref. [27] does not support the idea that the screening of the $\mathrm{CP}$ and the GE potential have the same origin and the same screening function can be used for both potentials as suggested in Ref. [33]. For that reason, here the GE potential without screening is taken, while in previous studies the small $\alpha_{\mathrm{V}}=0.30$ (a kind of screening) was used [15] and the GE potential with exponential screening function $\exp (-\delta r)(\delta=0.20 \mathrm{GeV})$ was taken in Ref. [16].

We also assume that the light mesons can be described by the universal GE potential (11) with the same parameters as in heavy quarkonia and heavy-light mesons [25,39], where the value of the vector coupling $\alpha_{\mathrm{V}}\left(n_{f}\right)$ is determined by the QCD vector constant $\Lambda_{\mathrm{V}}\left(n_{f}\right)$, which is defined through the QCD constant $\Lambda_{\overline{\mathrm{MS}}}\left(n_{f}\right)$ [41]:

$$
\Lambda_{\mathrm{V}}\left(n_{f}\right)=\Lambda_{\overline{\mathrm{MS}}}\left(n_{f}\right) \exp \left(-\frac{a_{1}}{2 \beta_{0}}\right)
$$

where $\beta_{0}=11-\frac{2}{3} n_{f}, a_{1}=\frac{31}{3}-\frac{10}{9} n_{f}$. For $n_{f}=3$ it gives

$$
\Lambda_{\mathrm{V}}\left(n_{f}=3\right)=1.4753 \Lambda_{\overline{\mathrm{MS}}}\left(n_{f}=3\right) .
$$

In $\mathrm{pQCD}$ the QCD constant $\Lambda_{\overline{\mathrm{MS}}}\left(n_{f}=3\right)=$ $0.339(10) \mathrm{GeV}$ is now known from the analysis of $\alpha_{s}\left(n_{f}\right)$, where the coupling $\alpha_{s}\left(M_{Z}\right)=0.1184(7)$ was taken as input and the matching procedure at the $b$-quark mass $\left(n_{f}=5\right)$ and the $c$-quark mass $\left(n_{f}=4\right)$ was performed [24]. Then the value $\Lambda_{\overline{\mathrm{MS}}}\left(n_{f}=3\right)=339(10) \mathrm{MeV}$ is obtained, which is significantly larger than that used in the past [3]. With the use of the relation (46) the value, $\lambda_{\mathrm{V}}\left(n_{f}=3\right)=500(15) \mathrm{MeV}$ follows and this large number has a small uncertainty. However, since the value $\Lambda_{\overline{\mathrm{MS}}}\left(n_{f}=3\right)$ depends on the $b$ - an $c$-quark masses, taken at the matching points, we expect that the uncertainty may be larger and this statement is confirmed in the analysis of the bottomonium spectrum [25], where a smaller $\Lambda_{\mathrm{V}}\left(n_{f}=3\right)=480(20) \mathrm{MeV}$ was shown to provide the best description of the bottomonium spectrum, if the IR regulator $M_{\mathrm{B}}=1.15 \mathrm{GeV}$ is used. Here in our study of the light meson spectra the preferable value of $\Lambda\left(n_{f}=3\right)$, which does not contradict the description of the bottomonium spectrum, is $\Lambda_{\mathrm{V}}\left(n_{f}=3\right)=460 \pm 20 \mathrm{MeV}$.

In coordinate space the strong vector coupling $\alpha_{\mathrm{V}}(r)\left(n_{f}=3\right)$ is expressed via the vector coupling $\alpha_{\mathrm{V}}\left(q^{2}\right)$ in the momentum space [16,25],

$$
\alpha_{\mathrm{V}}(r)=\frac{2}{\pi} \int_{0}^{\infty} \mathrm{d} q \frac{\sin (q r)}{q} \alpha_{\mathrm{V}}\left(q^{2}\right),
$$

which is taken in the two-loop approximation,

$$
\alpha_{\mathrm{V}}\left(q^{2}\right)=\frac{4 \pi}{9 t}\left(1-\frac{64}{81} \frac{\ln t}{t}\right)
$$

with $t=\ln \left[\left(q^{2}+M_{\mathrm{B}}^{2}\right) / \Lambda_{\mathrm{V}}^{2}\right]$. The parameters $\Lambda_{\mathrm{V}}$ and $M_{\mathrm{B}}$ are taken from Ref. [16],

$$
\begin{aligned}
\Lambda_{\mathrm{V}}\left(n_{f}=3\right) & =0.465 \mathrm{GeV}, \quad M_{\mathrm{B}}=1.15 \mathrm{GeV}, \\
\alpha_{\text {asym }} & =\alpha_{\mathrm{V}}(r \rightarrow \infty)=0.571, \quad \frac{4}{3} \alpha_{\text {asym }}=0.761 .
\end{aligned}
$$

where for $\Lambda_{\mathrm{V}}\left(n_{f}=3\right)=0.465 \mathrm{GeV}$ the frozen (asymptotic) coupling $\alpha_{\text {asym }}=0.571$, or $e_{\text {asym }}=0.761$, and just this value was used in our analysis of the RTs in Secs. III and IV. From Eq. (47) it can be derived that the asymptotic values of $\alpha_{\mathrm{V}}\left(q^{2}\right)$ and $\alpha_{\mathrm{V}}(r)$ coincide and the behavior of $\alpha_{\mathrm{V}}(r)$ for two values of the constant, $\Lambda_{\mathrm{V}}\left(n_{f}=3\right)=0.465 \mathrm{GeV}$ and $0.50 \mathrm{GeV}$, respectively, with $M_{\mathrm{B}}=1.15 \mathrm{GeV}$, is shown in Fig. 1 .

As seen from Fig. 1, the AF effect is important only for the $1 S, 1 P$, and $2 S$ states, whose sizes are $\lesssim 1.1 \mathrm{fm}$, and the following effective couplings $\left\langle\alpha_{\mathrm{V})}(r) r^{-1}\right\rangle_{n l}=\alpha_{\text {eff }}\left\langle r^{-1}\right\rangle_{n l}$ are defined:

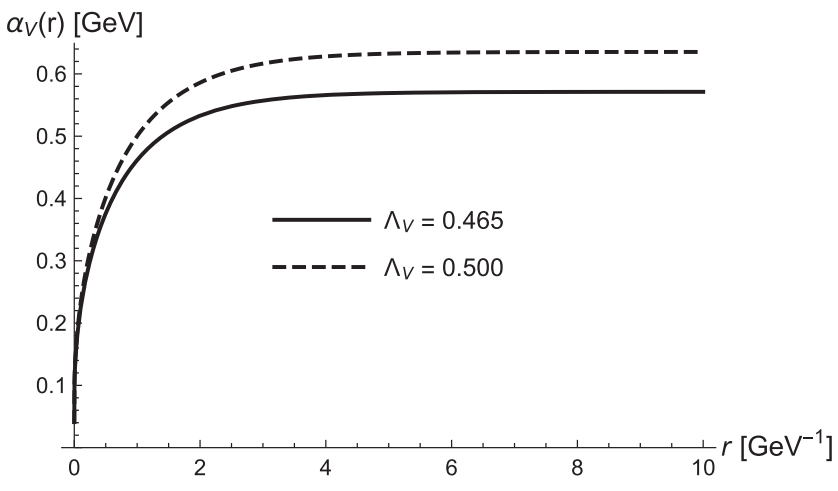

FIG. 1. The GE potential in $r$-space, for two values of $\Lambda_{\mathrm{V}}\left(n_{f}=3\right)$ : solid curve $\Lambda_{\mathrm{V}}=0.465 \mathrm{GeV}$, dashed curve $\Lambda_{\mathrm{V}}=0.500 \mathrm{GeV}$. 


$$
\begin{aligned}
\alpha_{\mathrm{eff}}(1 S) & =0.48, \quad e_{0}(1 S)=0.64, \\
\alpha_{\mathrm{eff}}(1 P) & =0.495, \quad e_{l}(1 P)=0.66, \\
\alpha_{\mathrm{eff}}(2 S) & =0.49 \quad(n \geq 1), \quad e_{0}(2 S)=0.65, \\
\alpha_{\mathrm{eff}} & =0.75(1) \quad(l \geq 2, n \geq 1) .
\end{aligned}
$$

As shown in Sec. III, the use of the effective coupling allows to present the physical picture in a clear way and for excited states provides the values of $\delta_{\mathrm{GE}}(n l)$, given in Table III, with accuracy $\sim(10-15) \mathrm{MeV}$.

\section{THE FLATTENED POTENTIAL $V_{\mathrm{f}}(r)$}

In the flattened $\mathrm{CP} V_{\mathrm{f}}(r)$ the string tension depends on $r$,

$$
V_{\mathrm{f}}(r)=\sigma_{\mathrm{f}}(r) r, \quad \sigma_{\mathrm{f}}(r)=\sigma(1-\gamma f(r)),
$$

where $\sigma(r)$ is defined by three parameters: first, the characteristic distance $R_{0} \sim(1.2-1.4) \mathrm{fm}$, where the flattening effect starts and string breaking becomes possible; its value is taken from the lattice calculations [22]. The second parameter, $\gamma$, determines the derivative of $V_{\mathrm{f}}(r)$ and the asymptotic value of the string tension,

$$
\sigma_{\mathrm{f}}(r \rightarrow \infty)=\sigma(1-\gamma) .
$$

The variation of the parameter $\gamma$ in the range $(0.30-0.50)$ has confirmed the result of Ref. [16] that $\gamma=(0.40-0.45)$ provides the best description of the spectrum (see Fig. 2, where the flattened CP is shown for $\gamma=0.40$ ).

The third parameter, the constant $B$, enters the function $f(r)$,

$$
f(r)=\frac{\exp \left(\sqrt{\sigma}\left(r-R_{0}\right)\right)}{B+\exp \left(\sqrt{\sigma}\left(r-R_{0}\right)\right)}
$$

where $f\left(r=R_{0}\right)=(B+1)^{-1}$ at the point $r=R_{0}$ shows how fast the increasing function $f(r)$ is approaching its

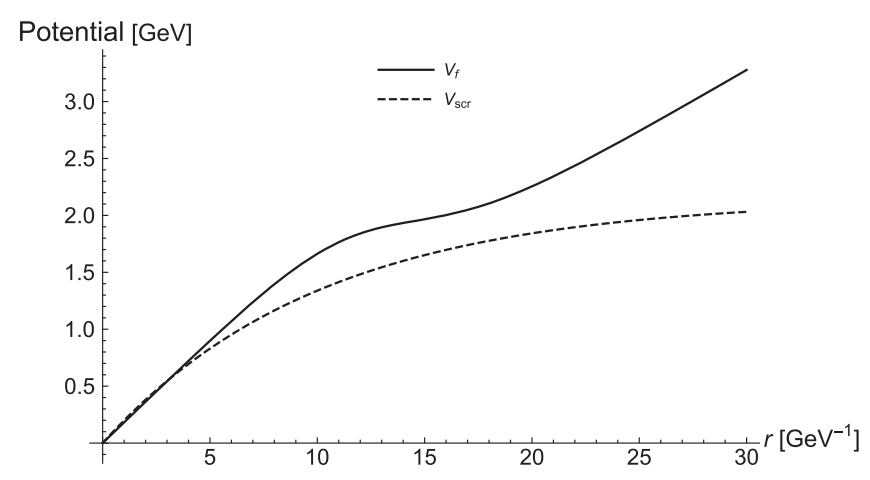

FIG. 2. The confining potential $V_{\mathrm{f}}(r)$ in $\mathrm{GeV}$ in $r$-space, $r$ in $\mathrm{GeV}^{-1}$ is shown with the parameters from Eq. (54), solid line, and the potential $V_{\text {scr }}(r)$ of $\mathrm{Li}$ and Chao, defined by Eq. (55), dashed line. asymptotic value, $f_{\text {asym }}=1.0$, at large distances, $r \sim 3.0 \mathrm{fm}$. In other aspects $f(r)$ can be rather arbitrary.

In our analysis all parameters were varied in wide ranges: $\gamma=0.35-0.50, B=15-25, R_{0}=(5-8) \mathrm{GeV}^{-1}=$ $(1.0-1.6) \mathrm{fm}$, for which $\sigma_{\mathrm{f}}($ asym $)=(0.090-0.11) \mathrm{GeV}^{2}$. The best description of the spectrum is reached for the parameter values

$$
\begin{aligned}
\gamma & =0.40, \quad B=20, \\
R_{0} & =6.0 \mathrm{GeV}^{-1}, \quad \sigma=0.182 \mathrm{GeV}^{2} .
\end{aligned}
$$

From the physical point of view it is important that at large distances the chosen potential, $V_{\mathrm{f}}(r) \rightarrow \sigma_{\mathrm{f} \text { asym }} r$ becomes again linear with small $\sigma_{\mathrm{f} \text { asym }}$ and therefore causes a quark and antiquark to be confined in a meson. This property of the flattened CP $V_{\mathrm{f}}(r)$ differs from that of a screened CP $V_{\text {scr }}(r)$, used in many papers $[33,34,42]$,

$$
\begin{aligned}
V_{\mathrm{scr}}(r) & =\lambda r F_{\mathrm{scr}}, \quad F_{\mathrm{scr}}=\frac{1-\exp (-\delta r)}{\delta r}, \\
\lambda & =0.21 \mathrm{GeV}^{2}, \quad \delta=0.0979 \mathrm{GeV},
\end{aligned}
$$

which has a linear behavior with large string tension $\lambda=$ $0.21 \mathrm{GeV}^{2}$ at $r<0.5 \mathrm{fm}$ and a screening effect that starts already at $r \sim 0.5 \mathrm{fm}$. At large distances $V_{\text {scr }}$ is approaching a constant, $V_{\text {scr }}($ asym $)=\frac{\lambda}{\delta}=2.145 \mathrm{GeV}$ and therefore the quark and antiquark inside a meson are not confined. The behavior of $V_{\text {scr }}(r)$ is compared with $V_{\mathrm{f}}(r)$ in Fig. 2. Notice that the screening function $F_{\text {scr }}$ cannot be used in the GE potential, since it produces the interaction $V_{\mathrm{GE}}(\mathrm{scr})=-\frac{e}{\delta} \frac{1-\exp (-\delta r)}{r^{2}}$, in which the main term, proportional to $r^{-2}$ with very large Coulomb constant $\frac{e}{\delta} \sim 5.0$, produces an unstable (unlimited) spectrum and cannot be used.

In Eq. (54) the string tension $\sigma=0.182 \mathrm{GeV}^{2}$ is chosen to keep for the $1 S$ state the averaged string tension, $\left\langle\sigma_{\mathrm{f}}(1 S)\right\rangle=0.180 \mathrm{GeV}^{2}$, as in the purely linear $\mathrm{CP}$. From our point of view the phenomenological potential $V_{\mathrm{f}}$ represents the physical picture rather well, but it has a negative feature, namely, the nonmonotonic behavior near the point $r=R_{0}$, and to obtain a smooth behavior of some matrix elements, a numerical regularization is needed.

With the flattened potential the spectrum significantly changes, as compared to the linear CP. First, the sizes $\sqrt{\left\langle r^{2}(n l)\right\rangle}$ of the excited states increase and can reach $\sim 2 \mathrm{fm}$ even for the $2 D$ and $3 S$ states (see Table V), although the sizes of the low states $(1 S, 1 P)$ remain not large, in particular, the r.m.s of the $\rho(1 S)$ meson, $\sqrt{\left\langle r^{2}(1 S)\right\rangle}=0.71 \mathrm{fm}$ (if the GE potential is present), is in good agreement with predictions in other approaches [43] and for these states the linear CP can be used. Secondly, due to the flattening effect the e.v.s $M_{0 \mathrm{f}}(n l)$ can be approximated like that in Eq. (21) and for the set of 
TABLE V. The r.m.s.(in fm) and $\left\langle r^{-1}(n l)\right\rangle$ (in $\mathrm{GeV}$ ) of light mesons $\left(m_{q}=0\right)$ for the linear potential (LP) $\left(\sigma=0.18 \mathrm{GeV}^{2}\right)$ and the flattened confining potential (FCP) with the parameters Eq. (54), and for the FCP + GE potential with the parameters from Eq. (49).

\begin{tabular}{lcccc}
\hline \hline State & r.m.s (LP) & r.m.s. FCP & r.m.s. FCP + GE & $\left\langle r^{-1}\right\rangle(\mathrm{FCP})$ \\
\hline $1 S$ & 0.82 & 0.86 & 0.71 & 0.357 \\
$2 S$ & 1.47 & 1.53 & 1.30 & 0.288 \\
$3 S$ & 1.65 & 2.42 & 2.12 & 0.204 \\
$4 S$ & 1.78 & 2.67 & 2.61 & 0.193 \\
$5 S$ & 2.08 & 2.94 & 2.79 & 0.189 \\
$1 P$ & 1.06 & 1.13 & 1.00 & 0.226 \\
$2 P$ & 1.43 & 1.95 & 1.69 & 0.176 \\
$3 P$ & 1.72 & 2.64 & 2.53 & 0.147 \\
$4 P$ & 1.97 & 2.78 & 2.69 & 0.146 \\
$1 D$ & 1.24 & 1.41 & 1.28 & 0.172 \\
$2 D$ & 1.56 & 2.42 & 2.18 & 0.134 \\
$3 D$ & 1.83 & 2.70 & 2.67 & 0.130 \\
$4 D$ & 2.06 & 2.94 & 2.83 & 0.127 \\
$1 F$ & 1.41 & 1.77 & 1.59 & 0.134 \\
$2 F$ & 1.70 & 2.73 & 2.61 & 0.115 \\
\hline \hline
\end{tabular}

parameters Eq. (54) and $\gamma=0.35,0.40,0.45$ the squared masses $M_{0 \mathrm{f}}^{2}(n l)$ with $n \geq 1$ can be presented as,

$$
\begin{aligned}
& M_{0 \mathrm{f}}^{2}(n l)\left(\mathrm{in} \mathrm{GeV}^{2}\right) \\
& \quad=0.86(5) l+1.24(10) n+2.51(10), \quad(\gamma=0.45), \\
& \quad=0.95(5) l+1.25(13) n+2.64(10), \quad(\gamma=0.40), \\
& \quad=1.04(8) l+1.35(8) n+2.65(6), \quad(\gamma=0.35) .
\end{aligned}
$$

In all these cases $n \geq 1$ and the ground states $(n=0)$ are not included, since their masses are determined by the linear $C P$. In Eq. (56) one can see that the orbital and radial slopes differ by $\sim 35 \%$ for all three values of $\gamma$, and the orbital slope $\beta_{l}$ and the radial slope $\beta_{n}$ increase with decreasing $\gamma$; also the values of both slopes are significantly smaller than $\beta_{l}=1.44 \mathrm{GeV}^{2}$ and $\beta_{n}=1.70 \mathrm{GeV}^{2}$ in the linear potential. This effect mostly occurs, because in the higher excitations the averaged $\langle\sigma(n l)\rangle$ is $\sim(20-30) \%$ smaller than the string tension $\sigma=0.18 \mathrm{GeV}^{2}$. It is of interest to notice that in the case with $\gamma=0.40$ calculated here, $\beta_{l}$ and $\beta_{n}$ turn out to be very close to the values obtained in the analysis of the experimental data in Refs. [11,12].

An unexpected result refers to the intercept, which in Eq. (56) is very large, $\cong 2.6 \mathrm{GeV}^{2}$, being even larger than in the linear CP. It means that in the flattened CP the GE and the SE corrections remain very important, while the string corrections are rather small. These corrections are defined by the same general formulas Eqs. (13), (14), if there the kinetic energy $\mu(n l)$ and $\sigma$ are replaced by the m.e.s $\left\langle\mu_{\mathrm{f}}\right\rangle$ and $\left\langle\sigma_{\mathrm{f}}\right\rangle$, respectively. However, the relations (19) and (20) for the m.e.s $\left\langle r^{-1}\right\rangle$ and $\mu_{\mathrm{f}}(n l)$ are not valid anymore and they have to be calculated in every case separately. In Table V we compare the r.m.s. in the linear $\mathrm{CP}$ with that in the flattened $\mathrm{CP}$ (FCP) and FCP + GE potential, and show that in the FCP, with or without the GE term, the sizes of the states with $n \geq 2$ strongly increase.

An interesting feature of $V_{\mathrm{f}}(r)$ refers to the averaged m.e.s $\left\langle r^{-1}(n l)\right\rangle$ (see Table V) and to $\left\langle\sigma_{\mathrm{f}}(n l)\right\rangle$ (see Table VI), which for excitations with $n>1$ coincide within $5 \%$ accuracy (if $l \geq 2$ ). Also, in the flattened $\mathrm{CP}$ the kinetic energies $\mu_{\mathrm{f}}(n l)$, as a function of $n$, grow slowly and have about $100-200 \mathrm{MeV}$ smaller values than $\mu_{0}(n l)$ in the linear CP. Due to this feature the self-energy correction, proportional to $\mu_{\mathrm{f}}^{-1}$, remains large, about -(240-300) MeV, and very important for high excitations. Also, in the presence of the GE potential the kinetic energy increases slowly, by $\sim(5-10) \%$, (see Table VII) and in some cases the difference between them can be neglected.

In the flattened $\mathrm{CP}$ the m.e.s $\left\langle r^{-1}\right\rangle_{n l}$ are small and practically equal (for high excitations), and therefore they cannot be expressed via the factors $A(n l)(20)$ and $Z(n l)$ (25). Moreover, these m.e.s are not proportional to the e.v.s $M_{0 \mathrm{f}}(n l)$ and this fact changes the physical picture. To calculate the GE correction the general form $\delta_{\mathrm{GE}}(n l)=$ $-e_{\text {eff }}\left\langle r^{-1}\right\rangle_{n l}$ has to be used, and in the string and the SE corrections the averaged string tensions, which are different in the states with different $l$ (and fixed $n$ ), have to be taken. Since the GE correction is small, the orbital and the radial slopes of the $M_{\mathrm{g}}^{2}(n l)$-trajectory, where $M_{\mathrm{g}}=M_{0 \mathrm{f}}+\delta_{\mathrm{GE}}$, practically do not change (see their values in Eq. (56) for $\gamma=0.40)$ :

$$
\begin{aligned}
M_{\mathrm{g}}^{2}(n l)\left(\mathrm{in} \mathrm{GeV}^{2}\right)= & 0.94(4) l+1.24(9) n+2.17(7) . \\
& (l \neq 0, \gamma=0.40, n \geq 1) .
\end{aligned}
$$

TABLE VI. The averaged $\left\langle\sigma_{\mathrm{f}}\right\rangle_{n l}$ (in $\mathrm{GeV}^{2}$ ) for the flattened CP with the parameters Eq. (54).

\begin{tabular}{lcccc}
\hline \hline$n / l$ & 0 & 1 & 2 & 3 \\
\hline 1 & 0.173 & 0.167 & 0.155 & 0.150 \\
2 & 0.162 & 0.165 & 0.150 & 0.148 \\
3 & 0.150 & 0.158 & 0.148 & 0.146 \\
\hline \hline
\end{tabular}

TABLE VII. The kinetic energies $\mu_{\mathrm{g}}(n l)$ (in $\mathrm{MeV}$ ) for the potential $V_{0 \mathrm{f}}(r)=V_{\mathrm{f}}(r)+V_{\mathrm{GE}}(r)$

\begin{tabular}{lcccc}
\hline \hline$n / l$ & 0 & 1 & 2 & 3 \\
\hline 0 & 400 & 491 & 539 & 564 \\
1 & 460 & 480 & 525 & 580 \\
2 & 520 & 482 & 536 & 594 \\
3 & 550 & 500 & 560 & 620 \\
\hline \hline
\end{tabular}


TABLE VIII. The eigenvalues $M_{0}(n S)$ (in MeV) of Eq. (7) with the linear potential, the shifts $\Delta_{f}(n S)=M_{0 \mathrm{f}}(n S)-M_{0}(n S)$, the corrections $\delta_{\mathrm{SE}}, \delta_{\mathrm{GE}}, \delta_{\mathrm{hf}}$, and the masses $M\left(n^{3} S_{1}\right)$.

\begin{tabular}{lccccccc}
\hline \hline State & $M_{0}(n S)$ & $\Delta_{\mathrm{f}}(n S)$ & $\delta_{\mathrm{SE}}(n S)$ & $\delta_{\mathrm{GE}}(n S)$ & $\delta_{\mathrm{hf}}(n S)$ & $M\left(n^{3} S_{1}\right)$ & $M(\exp )[10]$ \\
\hline $1^{3} S_{1}$ & 1339 & 0 & -405 & -225 & 66 & 775 & $\rho(775), M=775.5(3)$ \\
$2^{3} S_{1}$ & 1998 & -55 & -338 & -190 & 40 & 1455 & $\rho(1465), M=1465(25)$ \\
$3^{3} S_{1}$ & 2498 & -198 & -291 & -143 & 26 & 1892 & $\rho(1900), M=1880(30)$ \\
$4^{3} S_{1}$ & 2915 & -346 & -244 & -131 & 20 & 2214 & $\rho(2150), M=2254(22)$ \\
\hline \hline
\end{tabular}

Thus, in the flattened CP + GE potential the parameters of the $M_{\mathrm{g}}^{2}(n l)$-trajectory appear to be only $(1-3) \%$ smaller than those in the purely flattened CP.

In Tables VIII and IX besides the GE corrections, we give also the e.v.s $M_{0}(n l)$ of Eq. (7) with the linear $\mathrm{CP}$ and the mass shifts, produced by the flattening effect: $\quad \Delta_{\mathrm{f}}(n l)=M_{0}(n l)-M_{0 \mathrm{f}}(n l)$, which are large, $\sim-(300-350) \mathrm{MeV}$. As seen from Eq. (57), the masses $M_{\mathrm{g}}(n l)$, defined without the self-energy correction, are still $\sim(300-400) \mathrm{MeV}$ larger than their experimental values in Table IX and we come to the conclusion that the use of the flattened $\mathrm{CP}$ and the GE correction cannot provide the correct intercept, because the self-energy correction plays a dominant role to decrease its value.

In Table VIII the masses $M\left(n^{3} S_{1}\right)$ of the $n^{3} S_{1}$ states [for the set of the parameters (54)] together with the mass shifts due to the flattening effect, $\Delta_{\mathrm{f}}(n l)=M_{0 \mathrm{f}}(n l)-M_{0}(n l)$, and all corrections, including the hyperfine correction $\delta_{\mathrm{hf}}$, are given. The masses of the $n^{3} S_{1}$ states agree with the experimental values, with exception of the mass of $\rho(4 S)$, whose value is not well established yet [10]; notice, that the calculated mass, $M(\rho(4 S))=2214 \mathrm{MeV}$, is in agreement with the BABAR data [44]. Taking the masses from Table VIII, the slope $\beta_{n}(l=0)$ of the $\rho\left(n^{3} S_{1}\right)$ trajectory,

$$
\beta_{n}(l=0)=1.43(11) \mathrm{GeV}^{2},
$$

TABLE IX. The eigenvalues $M_{0}(n l)$ of Eq. (5) with linear CP, the mass shifts $\Delta_{f}(n l)$, the GE corrections $\delta_{\mathrm{GE}}(n l)$ (in $\mathrm{MeV}$ ) and $M_{\mathrm{g}}(n, l)=M_{0}+\Delta_{\mathrm{f}}+\delta_{\mathrm{GE}}$.

\begin{tabular}{lccccl}
\hline \hline State & $M_{0}(n l)$ & $\Delta_{\mathrm{f}}(n l)$ & $\delta_{\mathrm{GE}}(n l)$ & $M_{\mathrm{g}}(n l)$ & Exp. [10] \\
\hline $1 P$ & 1792 & 0 & -140 & 1652 & $a_{2}(1320)$ \\
$2 P$ & 2315 & -102 & -144 & 2069 & $a_{2}(1700)$ \\
$3 P$ & 2750 & -278 & -123 & 2349 & Absent \\
$4 P$ & 3129 & -398 & -114 & 2617 & Absent \\
$1 D$ & 2155 & 0 & -124 & 2031 & $\rho_{3}(1700)$ \\
$2 D$ & 2601 & -173 & -113 & 2315 & $\rho(1990)$ \\
$3 D$ & 2990 & -342 & -96 & 2552 & Absent \\
$4 D$ & 3337 & -448 & -94 & 2795 & Absent \\
$1 F$ & 2465 & 0 & -97 & 2368 & $a_{4}(2040)$ \\
$2 F$ & 2861 & -256 & -86 & 2519 & Absent \\
$3 F$ & 3215 & -394 & -84 & 2737 & Absent \\
\hline \hline
\end{tabular}

is obtained, which because of a large uncertainty can be considered to be approximately linear. Nevertheless, this radial slope is in good agreement with the experimental $\beta_{n}(l=0, \exp )=1.47(4) \mathrm{GeV}^{2}$, if the following experimental masses: $\rho(1 S)=775(3) \mathrm{MeV}, \quad \rho(2 S)=$ $1465(25) \mathrm{MeV}, \rho(3 S)=1890(20) \mathrm{MeV}$, and $\rho(4 S)=$ 2254 (22) MeV [10], are used.

The masses of the orbital excitations $M_{\mathrm{g}}(n l)$ are given in Table IX, where one can see that in the high excitations ( $l \neq 0, n \geq 2$ ) the shifts due to flattening, $\Delta_{\mathrm{f}} \sim-300 \mathrm{MeV}$, are very large, while the GE corrections, $\sim-90 \mathrm{MeV}$, are relatively small. However, without the SE corrections, the masses, $M_{\mathrm{g}}(n l)=M_{0}(n l)+\Delta_{\mathrm{f}}(n l)+\delta_{\mathrm{GE}}(n l),(l \geq 1, n \geq 1)$ exceed by $\sim(300-400) \mathrm{MeV}$ the experimental values.

\section{THE UNIVERSAL REGGE TRAJECTORIES}

In the previous section it was shown that in the flattened potential plus the GE correction, the masses $M_{\mathrm{g}}(n l)$ are larger than the experimental values by (300-400) MeV, and other corrections have to be taken into account. The string corrections $\delta_{\text {str }}$, defined by the Eq. (14), depend on the m.e.s $\left\langle r^{-1}\right\rangle_{n l}$, while the expressions, Eqs. (19) and (20), are not valid anymore and here the exact values of $\left\langle r^{-1}\right\rangle_{n l}$ from Table V (and the kinetic energy $\mu_{\mathrm{g}}(n l)$ from Table VII) are used. The values of the string correction,

$$
\delta_{\mathrm{str}}(n l)=-l(l+1)\langle\sigma(n l)\rangle \frac{\left\langle r^{-1}\right\rangle_{n l}}{8 \mu_{\mathrm{g}}^{2}(n l)} .
$$

are given in Table X. In high excitations they are small, $\sim-(30-50) \mathrm{MeV}$. On the contrary, the SE corrections remain large even in high excitations, where also the centroid masses $M_{\operatorname{cog}}(n l)$ are given. From this table one can also see that the centroid masses agree with the experimental masses, although the fine-structure splittings were not taken into account.

Then using the squared masses, the RT trajectory in the $\left(M_{\mathrm{cog}}^{2}, n l\right)$ plane $(l \neq 0)$ can be built,

$$
M_{\mathrm{cog}}^{2}(n, l)\left(\mathrm{in} \mathrm{GeV}^{2}\right)=1.03(9) l+1.15(9) n+0.65(15),
$$

where the orbital and the radial slopes have rather close values and even coincide within the theoretical errors. 
TABLE X. The centroid masses $M_{\mathrm{cog}}(n l)=M_{\mathrm{g}}(n l)+\delta_{\mathrm{SE}}+\delta_{\mathrm{str}}$ (in $\mathrm{MeV}$ ), calculated with the parameters, Eq. (49), and $\gamma=0.40$.

\begin{tabular}{lcccccl}
\hline \hline State & $M_{\mathrm{g}}(n l)$ & $\mu_{\mathrm{g}}(n l)$ & $\delta_{\mathrm{SE}}$ & $\delta_{\text {str }}$ & $M_{\mathrm{cog}}(n l)$ & Exp. [10] \\
\hline $1 P$ & 1652 & 491 & -330 & -40 & 1282 & $a_{2}(1320)$ \\
$2 P$ & 2069 & 480 & -310 & -33 & 1726 & $a_{2}(1700)$ \\
$3 P$ & 2349 & 482 & -291 & -30 & 2028 & Absent \\
$4 P$ & 2617 & 500 & -268 & -25 & 2324 & Absent \\
$1 D$ & 2031 & 539 & -292 & -77 & 1662 & $\rho_{3}(1690)$ \\
$2 D$ & 2315 & 525 & -262 & -60 & 1993 & $\rho_{3}(1990)$ \\
$3 D$ & 2552 & 536 & -257 & -51 & 2244 & Absent \\
$1 F$ & 2368 & 564 & -270 & -107 & 1991 & $a_{4}(2040)$ \\
$2 F$ & 2519 & 580 & -231 & -80 & 2208 & Absent \\
$3 F$ & 2737 & 594 & -221 & -74 & 2442 & Absent \\
\hline \hline
\end{tabular}

However, the central value of the orbital slope of this RT (60) is $\sim 10 \%$ smaller than that of the leading RT (43) and this difference illustrates the accuracy of our calculations using the flattened potential. In Table XI we compare the masses of the high excitations with $l \neq 0$, described by the RT (60), and those given in Table X. One can see that in most cases the agreement is better than $30 \mathrm{MeV}$.

We have chosen here the phenomenological flattened $\mathrm{CP}$ (51), but one cannot exclude that the true ("ideal") flattened $\mathrm{CP}$ is different, in particular, because in our case the matching of the linear and the flattened CP is not smooth. Therefore, equal values of the radial and orbital slopes are not excluded either. However, in our analysis the calculated RT can be called approximately universal.

It is important to stress that in the flattened CP plus the GE potential with the strong coupling, $\alpha_{\text {asym }}=0.57$, the masses of the high excitations are too large and only due to the self-energy correction correct values of the masses are obtained. Our analysis also has shown that in the flattened $\mathrm{CP}$ the role of the GE interaction is less important and therefore one cannot draw a definite conclusion whether at large distances a strong screening of the GE potential exists, or not. This statement is supported by our result that the RT for the flattened CP with $\gamma=0.40$ (56) and the RT (57), where in the masses the GE correction is taken into account, have practically equal $\beta_{l}=0.95(5) \mathrm{GeV}^{2}$ and radial slope, $\beta_{n} \sim 1.25(14) \mathrm{GeV}^{2}$.

To draw the conclusion whether the GE potential is screened or not, it is more perspicuous to study not very high excitations of light mesons, but to concentrate at lower resonances with $l=0,1$, whose sizes are not very large, $\sim 1 \mathrm{fm}$, and where the GE correction is more important. Also the information about the GE interaction at large distances can be extracted from the study of high excitations (with $n \geq 2$ ) in charmonium, or the bottomonium resonances above the $B \bar{B}$ threshold. Notice that in
TABLE XI. Comparison of the masses $M_{\text {cog }}$ (in $\mathrm{MeV}$ ) from Table $\mathrm{X}$ and those defined in the RT given in Eq. (60).

\begin{tabular}{lccl}
\hline \hline State & $M_{\mathrm{cog}}(n l)$ & $M(n l)$ Eq. $(60)$ & \multicolumn{1}{c}{ Exp. } \\
\hline $1 P$ & 1282 & 1315 & $a_{2}(1320)$ \\
$2 P$ & 1726 & 1697 & $a_{2}(1700)$ \\
$3 P$ & 2028 & 2007 & Absent \\
$4 P$ & 2324 & 2276 & Absent \\
$1 D$ & 1662 & 1661 & $\rho_{3}(1690)$ \\
$2 D$ & 1993 & 1977 & $\rho_{3}(1990)$ \\
$3 D$ & 2244 & 2249 & Absent \\
$1 F$ & 1991 & 1977 & $a_{4}(2000)$ \\
$2 F$ & 2244 & 2249 & Absent \\
$3 F$ & 2442 & 2467 & Absent \\
\hline \hline
\end{tabular}

charmonium the flattening effect is smaller than in light mesons [39], but the GE correction is larger.

\section{CONCLUSIONS}

The spectrum of light mesons was studied with the use of the RSH with the flattened confining potential (FCP), taking into account the gluon-exchange (GE), the selfenergy (SE), and the string corrections. We have confirmed that the flattening effect, existing due to the creation of light $q \bar{q}$-pairs, produces large mass shifts, which can reach $\sim-300 \mathrm{MeV}$ for the $3 P$ and $3 D$ excitations, and the best set of the flattened potential parameters was determined. Our calculations show that agreement with the experimental values of the masses can be reached, if all corrections are taken into account, but only the self-energy correction provides the linearity of the RT.

A special accent was placed on the role of the GE potential by performing calculations with the universal GE potential without screening, like the one that is used in heavy quarkonia. Our analysis has shown that for a weak GE potential (with strong screening) it is not possible to describe the leading $\operatorname{RT}(n=0)$, while the masses of high excitations weakly depend on the GE corrections. If the strong universal GE potential, as in heavy quarkonia, is taken, then the light meson masses with $l \neq 0$ are described by the RT, where the values of the orbital slope, $\beta_{l}=$ $1.03(9) \mathrm{GeV}^{2}$ and the radial slope, $\beta_{n}=1.15(12) \mathrm{GeV}^{2}$ are close, thus this RT can be considered as approximately universal and the predicted masses agree with the existing experimental data.

However, in any case the $n^{3} S_{1}$-trajectory does not belong to this RT, since their masses are strongly affected by the GE interaction and the spin-spin interaction, providing a large slope of the radial $\rho\left(n^{3} S_{1}\right)$ trajectory, $\beta_{n} \approx 1.43 \mathrm{GeV}^{2}$. 
[1] G. F. Chew and S. C. Frautschi, Phys. Rev. Lett. 7, 394 (1961); P. D. B. Collins, Phys. Rep. 1, 103 (1971).

[2] D. V. Bugg, Phys. Rep. 397, 257 (2004); E. Klempt and A. Zaitsev, Phys. Rep. 454, 1 (2007).

[3] S. Godfrey and N. Isgur, Phys. Rev. D 32, 189 (1985).

[4] D. Ebert, R. N. Faustov, and V. O. Galkin, Phys. Rev. D 79, 114029 (2009).

[5] A. V. Anisovich, V. V. Anisovich, and A. V. Sarantsev, Phys. Rev. D 62, 051502(R) (2000).

[6] V. V. Anisovich, Phys. Usp. 47, 45 (2004); A. V. Anisovich, D. V. Bugg, V. A. Nikonov, A. V. Sarantsev, and V. V. Sarantsev, Phys. Rev. D 85, 014001 (2012).

[7] D. V. Bugg, Phys. Rev. D 87, 118501 (2013).

[8] C. Amsler et al. (Crystal Barrel Collaboration), Eur. Phys. J. 23, 29 (2002).

[9] S. S. Afonin, Mod. Phys. Lett. A 22, 1359 (2007); Phys. Rev. C 76, 015202 (2007); S. S. Afonin and I. V. Pusenkov, Phys. Rev. D 90, 094020 (2014) and references therein.

[10] C. Patrignani et al. (PDG Collaboration), Chin. Phys. C 40, 100001 (2016).

[11] P. Masjuan, E. R. Arriola, and W. Broniowski, Phys. Rev. D 85, 094006 (2012).

[12] P. Masjuan, E. R. Arriola, and W. Broniowski, Phys. Rev. D 87, 118502 (2013).

[13] J. K. Chen, Phys. Lett. B 786, 477 (2018); D. Jia and W. C. Dong, arXiv:1804.08112.

[14] S. S. Afonin, Adv. High Energy Phys. 2019, 1701939 (2019); S. S. Afonin and T. D. Solomko, Int. J. Phys. A 33, 1850115 (2018).

[15] A. M. Badalian, B. L. G. Bakker, and Yu. A. Simonov, Phys. Rev. D 66, 034026 (2002).

[16] A. M. Badalian and B. L. G. Bakker, Phys. Rev. D 93, 074034 (2016).

[17] A. M. Badalian and B. L. G. Bakker, Phys. Rev. D 66, 034025 (2002).

[18] A. Yu. Dubin, A. B. Kaidalov, and Yu. A. Simonov, Phys. Lett. B 323, 41 (1994); Yad. Fiz. 56, 213 (1993) [Phys. At. Nucl. 56, 1745 (1993)].

[19] A. Yu. Dubin, A. B. Kaidalov, and Yu. A. Simonov, Phys. Lett. B 343, 310 (1995).

[20] V. L. Morgunov, A. V. Nefediev, and Yu. A. Simonov, Phys. Lett. B 459, 653 (1999).

[21] A. Duncan, E. Eichten, and H. Thacker, Phys. Rev. D 63, 111501(R) (2001); T. Kawanai and S. Sasaki, Phys. Rev. D 92, 094503 (2015) and references therein.

[22] G. S. Bali, Phys. Rev. D 62, 114503 (2000); Phys. Rep. 343, 1 (2001) and references therein.
[23] Yu. A. Simonov, Phys. Rev. D 99, 056012 (2019) and references therein.

[24] S. Bethke, Nucl. Phys. B, Proc. Suppl. 234, 229 (2013).

[25] A. M. Badalian and B. L. G. Bakker, Yad. Fiz. 77, 810 (2014) [Phys. At. Nucl. 77, 767 (2014)].

[26] A. Deau, S. J. Brodsky, and G. F. Teramond, Prog. Part. Nucl. Phys. 90, 1 (2016).

[27] M. S. Lukashov and Yu. A. Simonov, Phys. Rev. D 96, 076019 (2017).

[28] Yu. A. Simonov, Phys. Lett. B 515, 137 (2001); A. M. Badalian, Yu. A. Simonov, and V. I. Shevchenko, Phys. At. Nucl. 69, 1781 (2006) [A. M. Badalian, Yu. A. Simonov, and V. I. Shevchenko, Yad. Fiz. 69, 1818 (2006)].

[29] A. M. Badalian, B. L. G. Bakker, and I. V. Danilkin, Phys. Rev. D 81, 071502(R) (2010).

[30] S. J. Brodsky, Few-Body Syst. 57, 703 (2016).

[31] G. S. Bali, Phys. Rev. D 62, 114503 (2000).

[32] V. I. Shevchenko and Yu. A. Simonov, Phys. Rev. Lett. 85, 1811 (2000).

[33] P. Gonzalez, Phys. Rev. D 80, 054010 (2009); P. Gonzalez, V. Mathieu, and V. Vento, Phys. Rev. D 84, 114008 (2011) and references therein.

[34] B. Q. Li and K. T. Chao, Phys. Rev. D 79, 094004 (2009); B. Q. Li, C. Meng, and K. T. Chao, Phys. Rev. D 80, 014012 (2009).

[35] G. S. Bali, Phys. Lett. B 460, 170 (1999).

[36] A. M. Badalian, Phys. At. Nucl. 63, 2173 (2000); A. M. Badalian and D. S. Kuzmenko, Phys. Rev. D 65, 016004 (2001).

[37] Yu. A. Simonov, Phys. At. Nucl. 74, 1223 (2011).

[38] Yu. A. Simonov, Phys. At. Nucl. 68, 1294 (2005).

[39] A. M. Badalian, B. L. G. Bakker, and I. V. Danilkin, Phys. Rev. D 79, 037505 (2009); Phys. At. Nucl. 73, 138 (2010).

[40] Yu. A. Simonov, Nucl. Phys. B23, 283 (1991); Yu. S. Kalashnikova and D. S. Kuzmenko, Phys. At. Nucl. 66, 955 (2003).

[41] M. Peter, Phys. Rev. Lett. 78, 602 (1997); Y. Schroder, Phys. Lett. B 447, 321 (1999).

[42] L. C. Gui, L. S. Lu, Q. F. Lu, X. H. Zhong, and Q. Zhao, Phys. Rev. D 98, 016010 (2018); P. C. Ortega, J. Segovia, D. R. Entem, and F. Fernandez, Phys. Rev. D 94, 114018 (2016); X. H. Liu, arXiv:1602.01385.

[43] A. F. Krutov, R. G. Polezhaev, and V. E. Troitsky, Phys. Rev. D 93, 036007 (2016) and references therein.

[44] B. Aubert et al. (BABAR Collaboration), Phys. Rev. D 76, 092005 (2007). 